\title{
X-RAY AND SUNYAEV-ZEL'DOVICH EFFECT MEASUREMENTS OF THE GAS MASS FRACTION IN GALAXY CLUSTERS
}

\author{
Samuel J. LaRoque, ${ }^{1}$ Massimiliano Bonamente, ${ }^{2,3}$ John E. Carlstrom, ${ }^{1,4}$ Marshall K. Joy, ${ }^{3}$ \\ Daisuke Nagai, ${ }^{1,5}$ Erik D. Reese, ${ }^{6}$ and Kyle S. Dawson ${ }^{7,8}$ \\ Received 2006 April 4; accepted 2006 July 26
}

\begin{abstract}
We present gas mass fractions of 38 massive galaxy clusters at redshifts $0.14 \leq z \leq 0.89$, derived from Chandra $\mathrm{X}$-ray and OVRO/BIMA interferometric Sunyaev-Zel'dovich effect (SZE) measurements. We use three models for the gas distribution: (1) an isothermal $\beta$-model fit jointly to the X-ray data at radii beyond $100 \mathrm{kpc}$ and to all of the SZE data, (2) a nonisothermal double $\beta$-model in hydrostatic equilibrium with a Navarro-Frenk-White (NFW) dark matter distribution, fit jointly to all of the X-ray and SZE data, and (3) an isothermal $\beta$-model fit only to the SZE spatial data. We show that the isothermal model well characterizes the intracluster medium outside the cluster core and provides good fits to clusters with a range of morphological properties. X-ray and SZE mean gas mass fractions for model 1 are $f_{\text {gas }}(\mathrm{X}$-ray $)=0.110 \pm 0.003_{-0.018}^{+0.006}$ and $f_{\text {gas }}(\mathrm{SZE})=0.116 \pm 0.005_{-0.026}^{+0.009}$ assuming $\left(\Omega_{M}, \Omega_{\Lambda}, h\right)=$ $(0.3,0.7,0.7)$; uncertainties are statistical followed by systematic at $68 \%$ confidence. For model $2, f_{\text {gas }}(\mathrm{X}$-ray $)=$ $0.119 \pm 0.003_{-0.014}^{+0.007}$ and $f_{\text {gas }}(\mathrm{SZE})=0.121 \pm 0.005_{-0.016}^{+0.009}$. For model $3, f_{\mathrm{gas}}(\mathrm{SZE})=0.120 \pm 0.009_{-0.027}^{+0.009}$. The agreement in the results shows that the core can be accounted for satisfactorily by either excluding it from fits to the X-ray data or modeling the intracluster gas with a nonisothermal double $\beta$-model. We find that the SZE is largely insensitive to core structure. Our results indicate that the ratio of gas mass fraction within $r_{2500}$ to the cosmic baryon fraction, $f_{\text {gas }} /\left(\Omega_{B} / \Omega_{M}\right)$, is $0.68_{-0.16}^{+0.10}$, where statistical and systematic uncertainties are included at $68 \%$ confidence. By assuming that cluster gas mass fractions are independent of redshift, we find that the results agree with standard $\Lambda \mathrm{CDM}$ cosmology and are inconsistent with a flat matter-dominated $\left(\Omega_{M}=1\right)$ universe.
\end{abstract}

Subject headings: cosmological parameters - cosmology: observations - X-rays: galaxies: clusters

Online material: color figures

\section{INTRODUCTION}

Massive galaxy clusters $\left(M \sim 10^{15} M_{\odot}\right)$ are thought to be the relatively recent descendants of rare high-density fluctuations in the primordial universe. The evolution of massive clusters is critically dependent on cosmology, in particular on the matter density $\Omega_{M}$ and the normalization of the power spectrum $\sigma_{8}$. Furthermore, as massive galaxy clusters have collapsed from volumes of the order of $1000 \mathrm{Mpc}^{3}$, their composition should reflect that of the nonrelativistic components of the universe; i.e., the baryon budget of a cluster should reflect $\Omega_{B} / \Omega_{M}$.

For these reasons there is considerable interest in using cluster observations to constrain cosmology, and there has been significant success. For example, early X-ray observations were used to constrain the gas mass fraction of clusters and thereby set a lower limit to $\Omega_{B} / \Omega_{M}$. Using constraints on $\Omega_{B}$ from big bang nucleosynthesis, this led to upper limits on $\Omega_{M}$ that strongly ruled out a flat matter-dominated universe (White et al. 1993); a precise measurement of $\Omega_{M}$ was not possible due to insufficient understanding of the cluster baryon budget. A low $\Omega_{M}$ was also indicated by the

\footnotetext{
${ }^{1}$ Kavli Institute for Cosmological Physics, Department of Astronomy and Astrophysics, University of Chicago, 5640 South Ellis Avenue, Chicago, IL 60637.

2 Department of Physics, University of Alabama, Huntsville, AL 35812.

3 NASA Marshall Space Flight Center, Huntsville, AL 35812.

4 Department of Physics, Enrico Fermi Institute, University of Chicago, Chicago, IL 60637 .

5 Theoretical Astrophysics, California Institute of Technology, Mail Code 13033, Pasadena, CA 91125.

${ }^{6}$ Department of Physics, University of California, Davis, CA 95616.

7 Department of Physics, University of California, Berkeley, CA 94720.

8 Current address: Lawrence Berkeley National Laboratory, Berkeley, CA 94720.
}

discovery of high-redshift $(z \sim 1)$ massive clusters, which should be extremely rare in a matter-dominated, flat universe (Bahcall \& Fan 1998; Donahue et al. 1998).

Cosmological constraints can be obtained by exploiting the expected redshift independence of cluster gas mass fractions; only if the correct cosmology is used in the derivation of the gas mass fractions for a sample of clusters spanning a reasonable redshift range would the resulting gas mass fractions be constant with redshift (Sasaki 1996; Pen 1997). This technique is independent of the uncertainty in the cluster baryon budget, as well as that in the value of the Hubble constant.

Subsequently there have been many more cosmological studies using cluster X-ray and Sunyaev-Zel'dovich effect (SZE) measurements (e.g., see White et al. 1993; David et al. 1995; Evrard 1997; Myers et al. 1997; Mohr et al. 1999; Ettori \& Fabian 1999; Grego et al. 2001; Allen et al. 2002, 2004; Sanderson \& Ponman 2003). In general the results are in good agreement, along with cosmic microwave background (CMB; i.e., Spergel et al. 2006) and Type Ia supernova measurements (Perlmutter et al. 1999; Riess et al. 1998), with the now standard $\Lambda$ CDM cosmology.

Future large-scale cluster surveys are being pursued that will exploit the critical cosmological dependence of the evolution of cluster abundance to provide independent, precise determinations of $\Omega_{M}, \Omega_{\Lambda}, \sigma_{8}$, and the equation of state of dark energy, $w(z)$ (e.g., Kneissl et al. 2001; Romer et al. 2001; Kosowsky 2003; Ruhl et al. 2004). The ability to extract cosmology from large surveys using SZE, X-rays, or any other cluster observable will depend on the ability to link the measurements to cluster mass. An improved understanding of cluster structure and evolution is of interest in itself and would clearly also be helpful for improving cosmological measurements with clusters. 
In this paper we investigate the gas mass fractions for a sample of 38 clusters spanning redshifts from 0.14 to 0.89 , using Chandra $X$-Ray Observatory data and Berkeley-Illinois-Maryland Association (BIMA) and Owens Valley Radio Observatory (OVRO) interferometric radio SZE data. This SZE cluster sample is the largest yet compiled by over a factor of 2 . By combining the SZE data with the high-resolution Chandra X-ray data we are able to compare the SZE and X-ray gas mass fractions using two different models for the intracluster medium (ICM). Comparison of the SZE and X-ray results allows us to place constraints on the possible systematic effects associated with our incomplete knowledge of cluster properties. The results also provide constraints on the cluster baryon budget and thereby provide an important observational benchmark for cluster simulations. The baryon budget is sensitive to the details of cluster formation, gas cooling, and star formation history of the ICM (Ettori et al. 2006). Finally, we consider the cosmological constraints derived by assuming the cluster gas mass fractions of our sample do not evolve with redshift.

The paper is organized as follows: we review the theory underlying the X-ray emission and the SZE in clusters in $\S 2$ and describe the X-ray and SZE data in $\S 3$. In $\S 4$ we describe the cluster models and analysis methods used to determine the gas mass fractions, and we present tests of these models. In $\S 5$ we discuss additional sources of uncertainty in the gas mass fraction measurements, both statistical and systematic. The results are presented in $\S 6$, and constraints on the cluster baryon budget and cosmological parameters are detailed in $\S 7$. Finally, we summarize our conclusions in $\S 8$. All uncertainties are at the $68.3 \%$ confidence level, and a $\Lambda$ CDM cosmology with $\Omega_{M}=0.3, \Omega_{\Lambda}=$ 0.7 , and $h=0.7$ is assumed unless otherwise stated.

\section{X-RAY EMISSION AND SUNYAEV-ZEL'DOVICH EFFECT}

$\mathrm{X}$-ray emission in clusters arises predominantly from thermal bremsstrahlung for gas electron temperatures $T_{e} \gtrsim 3 \mathrm{keV}$. The $\mathrm{X}$-ray emissivity (typical cgs units ergs s $\mathrm{s}^{-1} \mathrm{~cm}^{-3} \mathrm{~Hz}^{-1}$ ) is not a directly observable quantity; instead X-ray proportional counters and CCDs measure the X-ray surface brightness over some frequency band,

$$
S_{\mathrm{X}}=\frac{1}{4 \pi(1+z)^{4}} \int n_{e} n_{\mathrm{H}} \Lambda_{e \mathrm{H}} d l
$$

where the integral is along the line of sight and $\Lambda_{e \mathrm{H}}$ is called the X-ray cooling function (e.g., Sarazin 1988) and is proportional to $T_{e}^{1 / 2}$. The observed X-ray emission can also be used to measure the gas temperature using instruments with spectral capability.

The cooling function is a proportionality factor relating the $\mathrm{X}$-ray emissivity to the ion and electron densities of the gas. Since $n_{i}$ and $n_{e}$ are related by the molecular weight of the gas and since electron-ion bremsstrahlung is the dominant emission process above $3 \mathrm{keV}$ [with emissivity per unit frequency $\epsilon_{\nu}^{\text {brem }} \propto$ $\left.T_{e}^{-1 / 2} n_{e} n_{i} \exp \left(-h \nu / k_{\mathrm{B}} T_{e}\right)\right]$, the cooling function in hot clusters $\Lambda_{e \mathrm{H}} \propto n_{e}^{2} T_{e}^{1 / 2}$, where we have integrated over the frequency band to which the instrument is sensitive. Calculation of $\Lambda_{e \mathrm{H}}$ from the $\mathrm{X}$-ray data is described in $\S 4.2$.

The thermal SZE is a small $(\lesssim 1 \mathrm{mK})$ distortion in the CMB spectrum caused by inverse Compton scattering of the CMB photons off of energetic electrons in the hot intracluster gas (Sunyaev \& Zel'dovich 1970, 1972). The spectral distortion can be expressed for dimensionless frequency $x \equiv h \nu / k_{\mathrm{B}} T_{\mathrm{CMB}}$ as a temperature change $\Delta T$ relative to the CMB temperature $T_{\mathrm{CMB}}$ :

$$
\frac{\Delta T}{T_{\mathrm{CMB}}}=f(x) y=f(x) \int \sigma_{\mathrm{T}} n_{e} \frac{k_{\mathrm{B}} T_{e}}{m_{e} c^{2}} d l,
$$

where $y$ is the Compton $y$-parameter, $\sigma_{\mathrm{T}}$ is the Thomson scattering cross section of the electron, $f(x)$ contains the frequency dependence of the SZE, and the integral is along the line of sight. The frequency dependence can be expressed as

$$
f(x)=\left(x \frac{e^{x}+1}{e^{x}-1}-4\right)\left[1+\delta_{\mathrm{SZE}}\left(x, T_{e}\right)\right],
$$

where $\delta_{\mathrm{SZE}}\left(x, T_{e}\right)$ is a relativistic correction. We adopt the analytic corrections of Itoh et al. (1998), which are good to fifth order in $k_{\mathrm{B}} T_{e} / m_{e} c^{2}$. The linear density and temperature dependences of the SZE make it a complementary probe to the X-ray emission, which varies as $n_{e}^{2} T_{e}^{1 / 2}$. The SZE is a decrement at low frequencies $(\lesssim 218 \mathrm{GHz})$ and an increment at high frequencies due to the upscattering of photons by the hot electrons. There is also a kinetic SZE that arises from scattering of CMB photons in a cluster with line-of-sight motion relative to the CMB rest frame. Discussion of the SZE in this work refers to the thermal SZE unless otherwise specified. We address the possible effects of the kinetic SZE on our results in $\S 5$.

With knowledge of the gas temperature, one can use either $S_{\mathrm{X}}$ or $\Delta T$ to determine the gas distribution and obtain $M_{\text {gas }}$. The total mass $M_{\text {total }}$ can be determined by assuming that the gas is in hydrostatic equilibrium (HSE) with the cluster potential. The gas mass fraction $f_{\text {gas }}$ is then $M_{\text {gas }} / M_{\text {total }}$.

\section{DATA}

The cluster sample consists of clusters that have both X-ray data from the Chandra X-Ray Observatory and SZE data from the BIMA/OVRO SZE imaging project, which uses the BerkeleyIllinois-Maryland Association (BIMA) and Owens Valley Radio Observatory (OVRO) interferometers to image the SZE. The BIMA/OVRO SZE imaging project has generally pursued hot clusters (published $T_{\mathrm{X}} \gtrsim 5 \mathrm{keV}$ ) that by inference should be massive and have a strong SZE. Properties of the cluster sample, including full cluster names, redshifts, positions, and Chandra and BIMA/OVRO observing information, are listed in Table 1.

The X-ray data were reduced using the Chandra Interactive Analysis of Observations (CIAO), version 3.2 software package with CALDB, version 3.1. Details of the data reduction procedure are given in Bonamente et al. (2004, 2006); briefly, CIAO and CALDB are used to apply corrections for charge transfer inefficiency (CTI) and buildup of a contaminant on the optical blocking filter. The observations are filtered to contain only photons with energies between 0.7 and $7.0 \mathrm{keV}$ and to remove contamination from solar flares. The data are then binned into images for each chip relevant to the observation, and X-ray backgrounds are extracted from appropriate cluster-free regions of the chips (Bonamente et al. 2004). Exposure maps are also created with CIAO, and all spatial analysis is performed on the binned images and exposure maps. Cluster spectra are extracted from a circular region centered on the cluster containing roughly $95 \%$ of the cluster counts, again restricted to the energy range $0.7-$ $7.0 \mathrm{keV}$. Spectra are grouped so that Gaussian statistics can be used in the spectral fitting, and a background spectrum is also extracted using the same background regions used in the image analysis. The software package XSPEC (Arnaud 1996) is used 
TABLE 1

Cluster Data

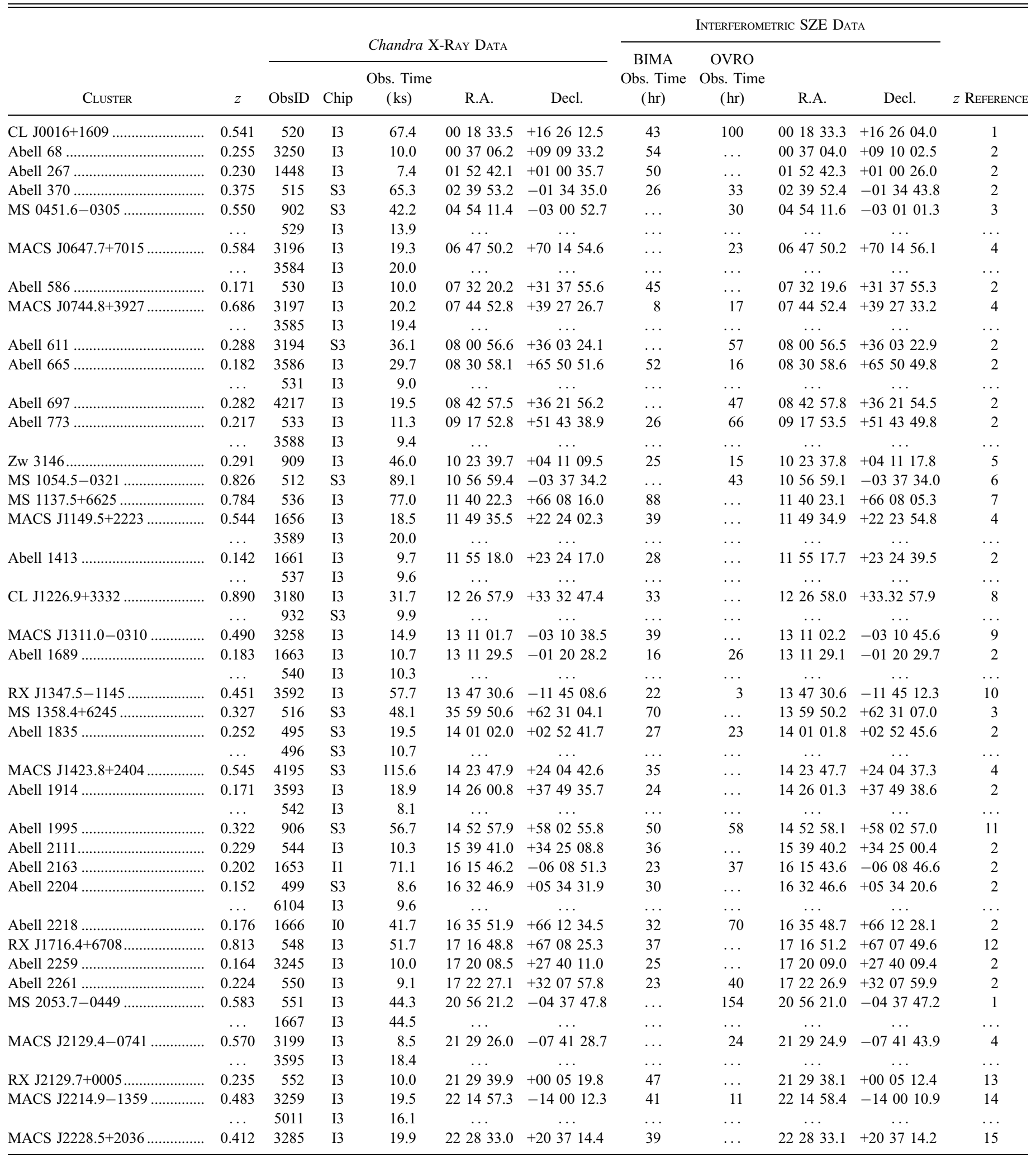

Noте.-Units of right ascension are hours, minutes, and seconds, and units of declination are degrees, arcminutes, and arcseconds.

References.- (1) Stocke et al. 1991; (2) Struble \& Rood 1999; (3) Gioia \& Luppino 1994; (4) LaRoque et al. 2003; (5) Allen et al. 1992; (6) Luppino \& Gioia 1995; (7) Donahue et al. 1999; (8) Ebeling et al. 2001; (9) Allen et al. 2004; (10) Schindler et al. 1995; (11) Patel et al. 2000; (12) Henry et al. 1997; (13) Ebeling et al. 1998; (14) this paper (derived from the Fe lines in the Chandra X-ray spectrum); (15) Böhringer et al. 2000. 
to fit a model to the spectrum and determine the X-ray spectroscopic temperature and metallicity of the cluster.

Interferometric radio observations of the cluster SZE were performed at the BIMA observatory and at OVRO. The millimeterwave arrays were equipped with $26-36 \mathrm{GHz}$ receivers for the SZE observations (Carlstrom et al. 1996). Most of the OVRO and BIMA telescopes were placed in a compact configuration to provide sensitivity on angular scales subtended by distant clusters (typically $\sim 1^{\prime}$ ), and a few telescopes were placed at longer baselines for simultaneous point-source imaging (Reese et al. 2002).

The SZE data consist of the position in the Fourier domain $(u-v$ plane) and the visibilities - the real and imaginary Fourier component pairs as functions of $u$ and $v$, which are the Fourier conjugate variables to right ascension and declination. The effective resolution of the interferometer, the synthesized beam, depends on the $u-v$ coverage and is therefore a function of the array configuration and source position. A typical size for the synthesized beam for the short baseline data is $\sim 1^{\prime}$. Details of the SZE data reduction can be found in Grego et al. (2001) and Reese et al. (2002). Briefly, the SZE data were reduced using the MIRIAD (Sault et al. 1995) and MMA (Scoville et al. 1993) software packages. Absolute flux calibration was performed using Mars observations adopting the brightness temperature from the Rudy (1987) Mars model. The gain was monitored with observations of bright radio point sources and remained stable at the $1 \%$ level over a period of months. Data were excised when one telescope was shadowed by another, when cluster observations were not bracketed by two phase calibrators, when there were anomalous changes in the instrumental response between calibrator observations, or when there was spurious correlation.

Images were made with the DIFMAP software package (Pearson et al. 1994) to inspect the data quality and, using only long baseline data, to identify and fix the positions of radio point sources. The point-source fluxes are included as free parameters in the model fitting, using the same methodology as in Reese et al. (2002).

\section{ANALYSIS METHODS AND MODELING}

\subsection{Cluster Density Models}

The isothermal $\beta$-model has frequently been used in the analysis of X-ray and SZE cluster images (Cavaliere \& Fusco-Femiano 1976; Fusco-Femiano 1978; Jones \& Forman 1984; Elbaz et al. 1995; Grego et al. 2001; Reese et al. 2002; Ettori et al. 2004). The three-dimensional (3D) electron number density is given by

$$
n_{e}(\boldsymbol{r})=n_{e_{0}}\left(1+\frac{r^{2}}{r_{c}^{2}}\right)^{-3 \beta / 2},
$$

where $n_{e}$ is the electron number density, $r$ is the radius from the center of the cluster, $r_{c}$ is the core radius of the ICM, and $\beta$ is a power-law index. A convenient feature of the isothermal $\beta$-model is that the X-ray surface brightness and SZE decrement profiles take simple analytic forms,

$$
\begin{gathered}
S_{\mathrm{X}}=S_{\mathrm{X} 0}\left(1+\frac{\theta^{2}}{\theta_{c}^{2}}\right)^{(1-6 \beta) / 2}, \\
\Delta T=\Delta T_{0}\left(1+\frac{\theta^{2}}{\theta_{c}^{2}}\right)^{(1-3 \beta) / 2},
\end{gathered}
$$

where $S_{\mathrm{X} 0}$ is the central X-ray surface brightness, $\Delta T_{0}$ is the central thermodynamic SZE temperature decrement/increment, and $\theta_{c}$ is the angular core radius of the cluster.
However, recent deep X-ray Chandra observations and numerical simulations indicate that the $\beta$-model is not a good description in the outskirts $\left(r>1 r_{2500}-1.5 r_{2500}\right)$ of clusters (Borgani et al. 2004; Vikhlinin et al. 2006); to avoid biases associated with this effect, we compute masses enclosed within $r_{2500}$, the radius at which the mean enclosed mass density is equal to $2500 \rho_{\text {crit }}$. Results are not extrapolated beyond this radius. Incidentally, $r_{2500}$ is also the outer limiting radius at which both the Chandra (e.g., Allen et al. 2004) and BIMA/OVRO data (Grego et al. 2001) provide strong constraints on the ICM model.

In some clusters the isothermal $\beta$-model fails to provide a good description of the X-ray surface brightness observed in the cluster core. This is the case, for instance, in highly relaxed clusters with sharply peaked central X-ray emission. We have therefore developed two extensions of the isothermal $\beta$-model to overcome this limitation; we describe these new models and their application to the X-ray and SZE data below.

\subsubsection{The $100 \mathrm{kpc}$ Cut Model}

First we consider a single isothermal $\beta$-model fit to the $\mathrm{X}$-ray data with the central $100 \mathrm{kpc}$ excised. The $100 \mathrm{kpc}$ radius is a good compromise, as it is large enough to exclude the cooling region in cool-core clusters while keeping a sufficient number of photons to enable the mass modeling. The X-ray spectroscopic temperature is also determined using photons extracted from a radial shell between $100 \mathrm{kpc}$ and $r_{2500}$. This is referred to as the $100 \mathrm{kpc}$ cut model in the remainder of this work.

There is no simple way to excise the central $100 \mathrm{kpc}$ from the interferometric SZE data because these data are fit in the Fourier plane. We therefore fit the entire SZE data set, while using the $\mathrm{X}$-ray spectroscopic temperature from the $100 \mathrm{kpc}$ cut model. The inclusion of the dense core in the SZE data should have little effect on the derived cluster parameters because the SZE as a probe of pressure is less sensitive to behavior in the dense core than are the X-ray data. It should also have little effect on the bestfit shape parameters, $\theta_{c}$ and $\beta$, because these fits are driven mainly by the X-ray data. The SZE data therefore mainly constrain the overall normalization of the SZE signal, which is insensitive to the details of the cluster core (e.g., Nagai 2006).

\subsubsection{The Nonisothermal Double $\beta$-Model}

We also develop a more sophisticated cluster plasma model that takes into account temperature profiles. A motivation for considering this model is to assess the biases arising from the isothermal assumption and the effects of the core exclusion in the previous model.

The model uses a second $\beta$-model component to describe the sharply peaked X-ray emission present in the cores of some clusters (Mohr et al. 1999). The 3D density profile of the double $\beta$-model is expressed by

$$
n_{e}(\boldsymbol{r})=n_{e_{0}}\left[f\left(1+\frac{r^{2}}{r_{c_{1}}^{2}}\right)^{-3 \beta / 2}+(1-f)\left(1+\frac{r^{2}}{r_{c_{2}}^{2}}\right)^{-3 \beta / 2}\right]
$$

where the two core radii, $r_{c_{1}}$ and $r_{c_{2}}$, describe the narrow, peaked central density component and the broad, shallow outer density profile, respectively, and $f$ represents the fractional contribution of the narrow, peaked component to the central density $n_{e_{0}}(0 \leq$ $f \leq 1$ ). This model has enough freedom to simultaneously fit the X-ray surface brightness in the outer regions and the central emission excess seen in some clusters. We set $f=0$ (equivalent 
of the single $\beta$-model) if the reduced $\chi^{2}$ for such a fit is less than 1.5 .

The 3D temperature profile is modeled assuming that the ICM is in hydrostatic equilibrium with a NFW dark matter density distribution (Navarro et al. 1996, 1997),

$$
\frac{\rho_{\mathrm{DM}}(r)}{\rho_{\mathrm{crit}}(z)}=\frac{\delta_{c}}{\left(r / r_{s}\right)\left(1+r / r_{s}\right)^{2}},
$$

where $\rho_{\text {crit }}(z)$ is the critical density of the universe at redshift $z$, $r_{s}=r_{200} / c$ is the scale radius, $c$ is the concentration parameter of the dark matter, and $\delta_{c}$ is the characteristic overdensity of the halo and is related to the concentration parameter $c$. Using the best-fit $3 \mathrm{D}$ gas density model, we solve the hydrostatic equation iteratively for the $3 \mathrm{D}$ model temperature profile. This $3 \mathrm{D}$ temperature profile is then weighted by the X-ray cooling function and the square of the cluster density, convolved with the appropriate instrumental response, and integrated along the line of sight, yielding a projected temperature profile model. Finally, the projected temperature profile model is compared to the observed temperature profile extracted from the Chandra data.

Altogether, the model is parameterized by the combination of the ICM density model, $n_{e_{0}}, \theta_{c_{1}}, \theta_{c_{2}}, \beta$, and $f$; the concentration parameter of the dark matter, $c$; and the outer radius, $r_{200}$. This model is referred to as the nonisothermal $\beta$-model in the remainder of this work.

\subsubsection{The SZE-only Model}

We also adopt a third model, used to fit the SZE spatial data independently of the X-ray spatial data. We use the full SZE data set with no $100 \mathrm{kpc}$ exclusion but use the $100 \mathrm{kpc}$ cut X-ray spectral data to determine the gas mass fractions. The SZE spatial data alone do not have sufficient resolution to provide strong individual constraints on $\theta_{c}$ and $\beta$ (Grego et al. 2001), so we fix $\beta$ at the median value of 0.7 for our sample. Values of $\beta$ in our sample are almost entirely in the range $0.5-1.0$, with most clustered between 0.6 and 0.8 ; this range is also consistent with many independent studies using other cluster samples (e.g., Jones \& Forman 1999; Mohr et al. 1999; Ettori et al. 2004). To test the effect of changing $\beta$, we have repeated the analysis with $\beta$ fixed at 0.6 and 0.8 , finding that all changes in the parameters are small relative to the $68 \%$ statistical uncertainties. We refer to this model as the SZE-only model in the remainder of this work.

\subsection{Model Fitting and Likelihood Analysis}

We determine the best-fit values and confidence intervals of the model parameters using likelihood analysis based on a Markov chain Monte Carlo (MCMC) method. We work with log likelihoods for both the spatial and spectral data. For spatial fitting to $\mathrm{X}$-ray and SZE images, the log likelihoods can be written (Cash 1979; Reese et al. 2002)

$$
\ln \left(\mathcal{L}_{\text {X-ray }}\right)=\sum_{i}\left[D_{i} \ln \left(M_{i}\right)-M_{i}-\ln \left(D_{i} !\right)\right]
$$

for X-ray data (Poisson) and

$$
\ln \left(\mathcal{L}_{\mathrm{SZE}}\right)=\sum_{i}-\frac{1}{2}\left(\Delta R_{i}^{2}+\Delta I_{i}^{2}\right) W_{i}
$$

for SZE data (Gaussian), where $M_{i}$ and $D_{i}$ are the model prediction and data in pixel $i$ in the X-ray data, while $\Delta R_{i}^{2}$ and $\Delta I_{i}^{2}$ are the difference between the real and imaginary components of the SZE data and model at each point $i$ in the Fourier plane, and $W_{i}=1 / \sigma_{i}^{2}$ is a measure of the Gaussian noise. Calculation of the spectral log likelihood is done with XSPEC. The spatial and spectral log likelihoods are then added together to compute the joint log likelihood. Bonamente et al. (2006) describe the likelihood analysis for the nonisothermal double $\beta$-model; in that case the X-ray and SZE central densities were linked and $D_{A}$ was a free parameter in the fit. Our approach differs only in that $D_{A}$ is fixed for an assumed cosmology and the X-ray and SZE central densities are fit separately so that the individual mass results can be compared. We therefore describe the likelihood calculation here only in the context of the $100 \mathrm{kpc}$ cut and SZE-only models.

In the $100 \mathrm{kpc}$ cut case, the X-ray model is given by equation (5) plus a constant X-ray background, $B_{\mathrm{X}}$. The model has five free parameters, including the two-dimensional cluster position $\left(x_{c}, y_{c}\right)$, central surface brightness $S_{\mathrm{X} 0}$, and shape parameters $\theta_{c}$ and $\beta$. The background, $B_{\mathrm{X}}$, is determined from the data using a method described in Bonamente et al. (2004) and held constant during the model fitting. The model is calculated at each pixel position and then multiplied by the exposure map before being compared to the image. X-ray point sources and any obvious substructure are masked out, and the masked pixels are ignored in the likelihood calculation. Any pixels within $100 \mathrm{kpc}$ of the cluster center are also ignored. The Poisson log likelihood is then computed using equation (9).

The interferometric SZE data are analyzed directly in the Fourier plane, where the noise characteristics and spatial filtering of the interferometer are well understood. In both the $100 \mathrm{kpc}$ cut and SZE-only cases, the composite SZE model consists of the isothermal $\beta$-model and any point sources detected in the field. The parameters of interest are the two-dimensional cluster position, the central decrement $\Delta T_{0}, \theta_{c}, \beta$, and the positions and fluxes of the point sources. The SZE $\beta$-model plus point-source model are constructed in the image plane, multiplied by the primary beam, and fast Fourier transformed to the $u-v$ plane for comparison with the data. The Gaussian log likelihood is then calculated using equation (10).

$\mathrm{X}$-ray spectral likelihoods are calculated using $\chi^{2}$ information from XSPEC. A photoabsorbed Raymond-Smith thermal plasma model (Raymond \& Smith 1977) is fit to the 0.7-7.0 keV X-ray spectral data, with $\mathrm{H}$ I column density fixed at the value from Dickey \& Lockman (1990) and solar abundances from Feldman (1992). XSPEC is used to create a table of $T_{e}$ and metallicity $Z$-values versus log likelihood for the spectral model using the relation $\ln \mathcal{L} \propto-\frac{1}{2} \chi^{2}$.

This same spectral information is also used to determine $\Lambda_{e \mathrm{H}}$ for each combination of $T_{e}$ and $Z$. We calculate the cooling function with an external Raymond-Smith spectral emissivity code that includes the dominant contribution from relativistic electronion thermal bremsstrahlung as well as lesser contributions from electron-electron thermal bremsstrahlung, recombination, and twophoton processes. The relativistic corrections of Gould (1980) are used in the bremsstrahlung calculation. The cooling function is then redshifted to the detector frame, convolved with the telescope response as read out from XSPEC, and integrated over the 0.7-7.0 keV Chandra bandpass. See Reese et al. (2000) for a more detailed description of this code.

Best-fit values and confidence intervals for all spatial and spectral model parameters, as well as $\Lambda_{e \mathrm{H}}$, are determined using a MCMC method that efficiently handles the large number of parameters involved in the model fitting. Implementation of a MCMC method for determining the angular diameter distance to the cluster A611 from SZE and X-ray data is described in detail in Bonamente et al. (2004) including choice of parameter support and convergence and mixing tests. 
For the $100 \mathrm{kpc}$ cut model, we use the likelihood analysis based on the MCMC method and fit the SZE and X-ray data jointly; $\theta_{c}$ and $\beta$ are linked between X-ray and SZE data sets (Reese et al. 2000), and the X-ray spectral model is also included. Since the SZE data do not constrain $\theta_{c}$ and $\beta$ well individually, the X-ray data set drives the fit to these parameters while $\Delta T_{0}$ is allowed to find its best-fit value. The central $100 \mathrm{kpc}$ are excluded from the X-ray data but not from the SZE data. Since the data sets are independent, the X-ray and SZE spatial log likelihoods and spectral $\log$ likelihood are added to determine the joint likelihood for this model.

For the SZE-only model, the MCMC method is used to fit the SZE data to an isothermal $\beta$-model; $\beta$ is fixed at 0.7 while $\theta_{c}$ and $\Delta T_{0}$ are allowed to find their best-fit values. An X-ray spectral model with the central $100 \mathrm{kpc}$ excluded from the X-ray data is also included. Since the SZE and X-ray data sets are independent, the SZE spatial log likelihood and X-ray spectral log likelihood are added to determine the joint likelihood for this model.

\subsubsection{Gas Mass, Total Mass, and Gas Mass Fraction}

With the best-fit ICM model and X-ray temperature in hand, it is straightforward to compute the gas mass and total mass of the cluster. For the $\beta$-model, the enclosed gas mass is obtained by integrating the best-fit 3D gas density profile,

$$
M_{\mathrm{gas}}(r)=A \int_{0}^{r / D_{A}}\left(1+\frac{\theta^{2}}{\theta_{c}^{2}}\right)^{-3 \beta / 2} \theta^{2} d \theta,
$$

where $A=4 \pi \mu_{e} n_{e_{0}} m_{p} D_{A}^{3}$, and $\mu_{e}$, the mean molecular weight of the electrons, is determined from the X-ray spectral data. By assuming the isothermal gas temperature, we can compute the gas mass independently from both the X-ray and SZE data sets. For the X-ray data, the model central electron density $n_{e_{0}}$ can be expressed analytically as (Birkinshaw et al. 1991)

$$
n_{e_{0}}=\left[\frac{S_{\mathrm{X} 0} 4 \pi(1+z)^{4}\left(\mu_{\mathrm{H}} / \mu_{e}\right) \Gamma(3 \beta)}{\Lambda_{e \mathrm{H}} D_{A} \pi^{1 / 2} \Gamma(3 \beta-1 / 2) \theta_{c}}\right]^{1 / 2}
$$

For the SZE, the model central electron density can be expressed as (e.g., Grego et al. 2001)

$$
n_{e_{0}}=\frac{\Delta T_{0} m_{e} c^{2} \Gamma[(3 / 2) \beta]}{f_{\left(\mathrm{X}, T_{e}\right)} T_{\mathrm{CMB}} \sigma_{\mathrm{T}} k_{\mathrm{B}} T_{e} D_{A} \pi^{1 / 2} \Gamma[(3 / 2) \beta-1 / 2] \theta_{c}} .
$$

For the $100 \mathrm{kpc}$ cut model, we compute the gas mass using equation (11) by extrapolating the model into the cluster centers. For the nonisothermal $\beta$-model, the gas mass is obtained by integrating the best-fit central density over a distribution similar to equation (11) but that accounts for the additional density component. In this case $\mu_{e}$ is treated as a constant, as its value changes by only $0.3 \%$ over the radial range considered. In the SZE-only analysis, the gas mass is computed using equation (11) with model central density from equation (13).

The total mass, $M_{\text {total }}$, can be obtained by solving the hydrostatic equilibrium equation as

$$
M_{\mathrm{total}}(r)=-\frac{k r^{2}}{G \mu_{\mathrm{total}} m_{p}}\left[T_{e}(r) \frac{d n_{e}(r)}{d r}+n_{e}(r) \frac{d T_{e}(r)}{d r}\right] .
$$

Under the isothermal assumption, this reduces to the simple analytic form (e.g., Grego et al. 2001):

$$
M_{\mathrm{total}}(r)=\frac{3 \beta k T_{e}}{G \mu m_{p}} \frac{r^{3}}{r_{c}^{2}+r^{2}},
$$

which can be used to calculate total masses for both the $100 \mathrm{kpc}$ cut and SZE-only models. For the nonisothermal $\beta$-model, the temperature derivative in equation (14) is simple to compute numerically. We then compute X-ray and SZE gas mass fractions as $f_{\text {gas }}=M_{\text {gas }} / M_{\text {total }}$ for the sample of 38 clusters.

\subsection{Comparison of the Density Models Fit to X-Ray Data}

We now compare the results of the X-ray surface brightness modeling and the cluster parameters extracted using different ICM models. The primary goal of this comparison is to assess the effects of the isothermal assumption and different treatments of the cluster core.

Figure 1 shows the isothermal $\beta$-model, nonisothermal $\beta$-model, and $100 \mathrm{kpc}$ cut model as fit to both the non-cool-core cluster A1995 and the cool-core cluster A1835. The X-ray surface brightness profiles are background-subtracted and constructed using concentric annuli centered on the cluster (e.g., Bonamente et al. 2006). The model fitting is done to the entire two-dimensional cluster image. Table 2 lists the spatial $\left(\theta_{c}\right.$ and $\beta$ ), spectral (isothermal spectroscopic gas temperature $\left.T_{\mathrm{X}}\right)$, and inferred $\left(M_{\mathrm{gas}}, M_{\mathrm{total}}\right.$, and $f_{\text {gas }}$ ) cluster quantities obtained using these three ICM models. The radius $r_{2500}$ is computed using parameters from the $100 \mathrm{kpc}$ cut models. Temperature profiles and data points for the nonisothermal models can be found in Bonamente et al. (2006), who demonstrate that the spectroscopic data are well fit by the model temperature profiles.

In the case of A1995 (a non-cool-core cluster), we find that the results are largely insensitive to the chosen model. The surface brightness profiles appear well fit by all three models, and the derived gas masses, total masses, and gas mass fractions are in good agreement. Although $\theta_{c}$ and $\beta$ differ slightly, these parameters are sufficiently degenerate that the difference has a very small effect on the derived masses. These results illustrate that the simple $100 \mathrm{kpc}$ cut model works as well as the more sophisticated nonisothermal double $\beta$-model for the non-cool-core clusters.

Analysis of the cool-core cluster A1835, on the other hand, highlights the importance of a proper treatment of the cluster core. The most striking differences are the masses derived from the isothermal $\beta$-model versus those from the other two models. This illustrates the shortcomings of a brute force application of the isothermal $\beta$-model to cool-core clusters. The mass discrepancies can be attributed to a poorer fit to the surface brightness at $r>100 \mathrm{kpc}$; this arises because the fit is driven mainly by the extremely high signal-to-noise ratio data in the cluster core. A1835 and other cool-core clusters tend to have extremely small core radii and $\beta$-parameters when fit by a single isothermal $\beta$-model. We also find that the X-ray spectroscopic temperature, $T_{\mathrm{X}}$, is biased low when the core is included in the determination of $T_{\mathrm{X}}$, which has an additional impact on the total mass estimate of the cluster.

The models can be quantitatively compared using a goodnessof-fit analysis. Goodness of fit for the X-ray data is assessed using Monte Carlo simulations, following Winkler et al. (1995). For a given cluster, we construct the best-fit model and compare with the data to determine the fit statistic from equation (9). Poisson noise is then randomly added to the best-fit model, creating $10^{4}$ new realizations, and the fit statistic is calculated for each by comparing them with the original best-fit model. The fraction of simulations that give a lower fit statistic than that of the bestfit model compared to the data is called the "goodness" (e.g., Jonker et al. 2005), with values near 0.5 indicating a good fit to the data and values near 0 or 1 indicating a poor fit.

In the case of A1995, all three models (standard isothermal $\beta$, $100 \mathrm{kpc}$ cut, and nonisothermal) provide acceptable descriptions of the data, with respective goodness values of $0.416,0.427$, and 

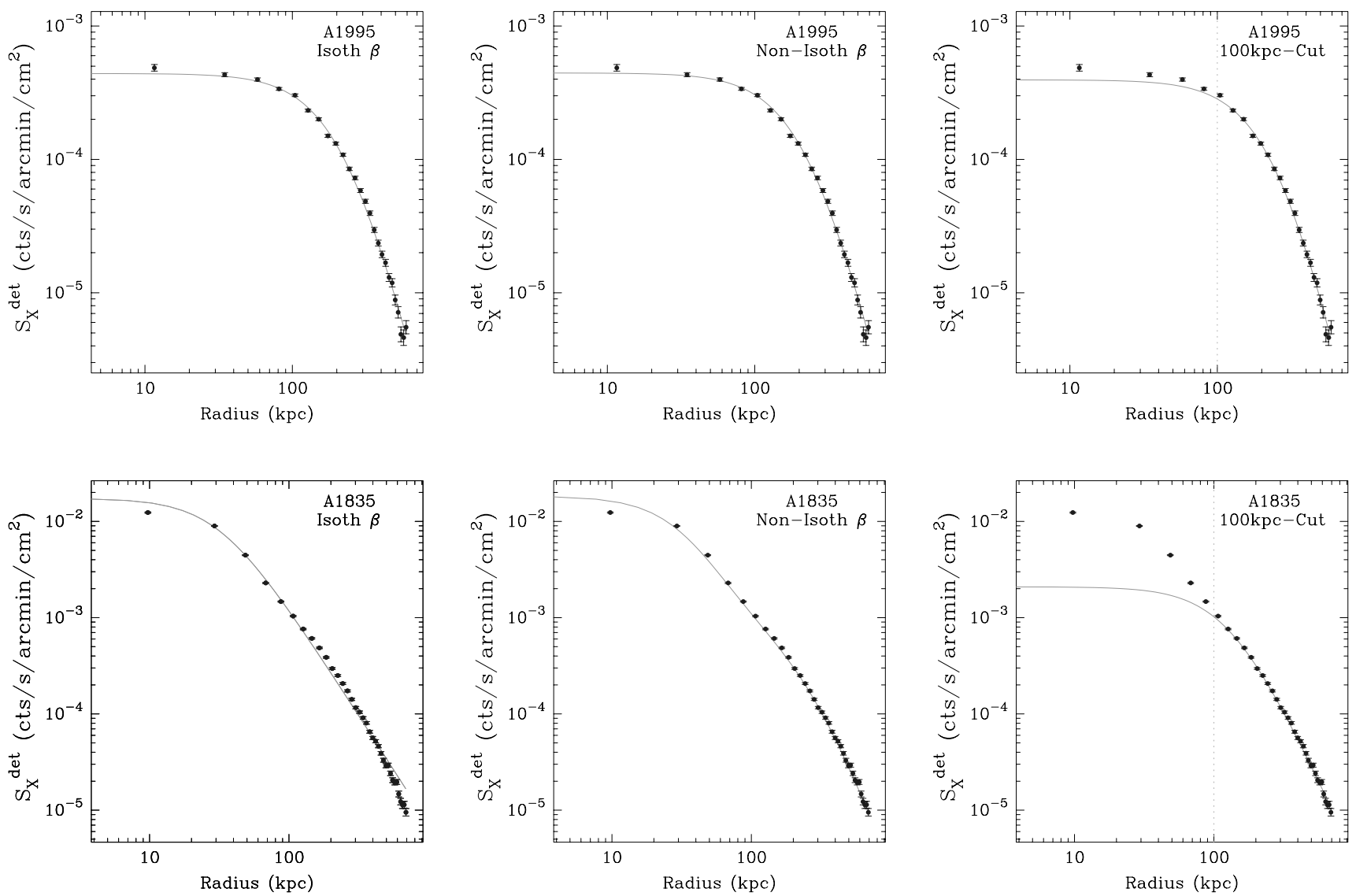

FIG. 1.-Comparison of X-ray surface brightness profiles for the isothermal $\beta$-model (left), nonisothermal $\beta$-model (middle), and $100 \mathrm{kpc}$ cut model (right) as applied to both the non-cool-core cluster A1995 (top) and the cool-core cluster A1835 (bottom). The dotted vertical line in the 100 kpc cut panels denotes the 100 kpc radius. [See the electronic edition of the Journal for a color version of this figure.]

0.496. Acceptable goodness values of $0.547,0.548$, and 0.603 , for the same three models, are also found for A1835. However, the goodness value for the standard isothermal $\beta$-model may be biased by the extremely high count rate in the core of this cluster. To investigate this, we use the best-fit parameters from the standard $\beta$-model but test them with the $100 \mathrm{kpc}$ cut data set. The result is a goodness of zero; the standard $\beta$-model is a poor description of the cluster gas distribution between $100 \mathrm{kpc}$ and $r_{2500}$. Tests on clusters in our sample indicate that $90 \%-95 \%$ of the cluster gas mass within $r_{2500}$ lies beyond a $100 \mathrm{kpc}$ radius (LaRoque
2005). It is therefore critical that the model give a good description of the data between $100 \mathrm{kpc}$ and $r_{2500}$, which the standard isothermal $\beta$-model fails to do. By contrast, when the best-fit nonisothermal $\beta$-model parameters are fit to the data between $100 \mathrm{kpc}$ and $r_{2500}$, the goodness is 0.776 , implying a much better description of the data in this region.

\subsection{Testing the Model with Simulations}

The ability of the $100 \mathrm{kpc}$ cut model to recover the gas mass can be further assessed by fitting the model to projected data from

TABLE 2

Comparison of Parameters for Different ICM Models

\begin{tabular}{|c|c|c|c|c|c|c|}
\hline Model & $\begin{array}{c}\theta_{c} \\
(\operatorname{arcsec})\end{array}$ & $\beta$ & $\begin{array}{c}T_{\mathrm{X}} \\
(\mathrm{keV})\end{array}$ & $\begin{array}{c}M_{\mathrm{gas}}\left(r_{2500}\right) \\
\left(10^{13} M_{\odot}\right)\end{array}$ & $\begin{array}{c}M_{\text {total }}\left(r_{2500}\right) \\
\left(10^{14} M_{\odot}\right)\end{array}$ & $f_{\text {gas }}$ \\
\hline \multicolumn{7}{|c|}{ A1995 } \\
\hline 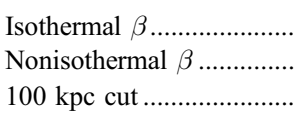 & $\begin{array}{c}50.3 \pm 1.5 \\
50.3_{-1.4}^{+1.5} \\
57.5_{-2.6}^{+2.7}\end{array}$ & $\begin{array}{c}0.921_{-0.023}^{+0.024} \\
0.916_{-0.023}^{+0.024} \\
1.01 \pm 0.04\end{array}$ & $\begin{array}{c}8.63_{-0.40}^{+0.39} \\
\cdots \\
8.22_{-0.45}^{+0.44}\end{array}$ & $\begin{array}{c}3.52 \pm 0.12 \\
3.79_{-0.23}^{+0.21} \\
3.51 \pm 0.14\end{array}$ & $\begin{array}{l}4.69 \pm 0.39 \\
4.42_{-0.71}^{+0.75} \\
4.74_{-0.48}^{+0.50}\end{array}$ & $\begin{array}{c}0.075 \pm 0.004 \\
0.086_{-0.009}^{+0.011} \\
0.074 \pm 0.005\end{array}$ \\
\hline \multicolumn{7}{|c|}{ A1835 } \\
\hline $\begin{array}{l}\text { Isothermal } \beta \ldots \ldots \ldots \ldots \ldots \ldots \\
\text { Nonisothermal } \beta \ldots \ldots \ldots \ldots \ldots . . .\end{array}$ & $\begin{array}{l}8.13 \pm 0.09 \\
9.08_{-0.21}^{+0.25 a} \\
61.6_{-1.8}^{+1.9 b} \\
33 .+10\end{array}$ & $\begin{array}{c}0.543 \pm 0.001 \\
0.784_{-0.014}^{+0.016}\end{array}$ & $\begin{array}{c}8.37 \pm 0.23 \\
\ldots \\
\ldots\end{array}$ & $\begin{array}{c}4.04 \pm 0.08 \\
6.49_{-0.33}^{+0.30}\end{array}$ & $\begin{array}{c}2.55 \pm 0.11 \\
5.77_{-0.71}^{+0.70}\end{array}$ & $\begin{array}{l}0.158_{-0.003}^{+0.004} \\
0.112_{-0.009}^{+0.009}\end{array}$ \\
\hline $100 \mathrm{kpc}$ cut ........................... & $33.6 \pm 1.0$ & $0.690_{-0.008}^{+0.007}$ & $11.4_{-0.6}^{+0.7}$ & $5.78_{-0.20}^{+0.23}$ & $5.56_{-0.42}^{+0.50}$ & $0.104 \pm 0.005$ \\
\hline
\end{tabular}

${ }^{\text {a }}$ Inner $\theta_{c}$.

${ }^{\mathrm{b}}$ Outer $\theta_{c}$ 

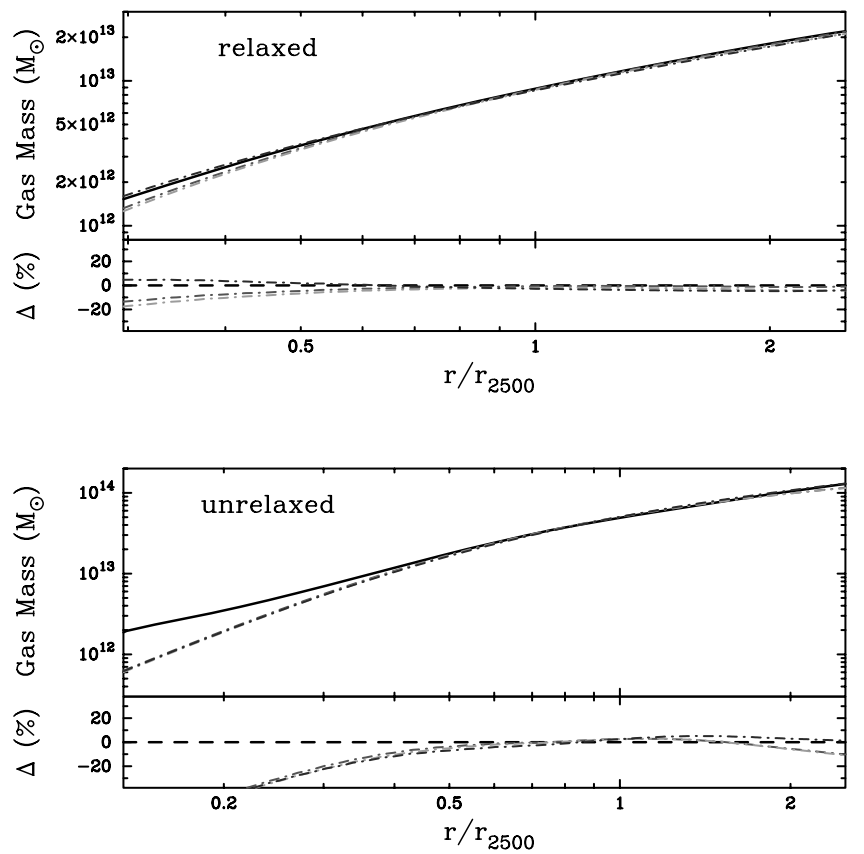

FIG. 2.-Ability of the $100 \mathrm{kpc}$ cut model to recover the gas mass at $r_{2500}$ in two simulated clusters, one highly relaxed (top) and one highly unrelaxed (bottom). The top part of each panel shows the true gas mass profile (black solid line) with dash-dotted lines showing profiles recovered from fitting the model to $x$-, $y$-, and $z$-projections. The bottom part of each panel shows the fractional deviation of recovered mass from true mass as a function of radius. [See the electronic edition of the Journal for a color version of this figure.]

simulated clusters with precisely known gas masses. This test is carried out using cosmological simulations of galaxy clusters generated with the Adaptive Refinement Tree (ART) $N$-body + gas dynamics code (Kravtsov 1999; Kravtsov et al. 2002). These simulations include radiative cooling and UV heating, star formation, and stellar feedback processes in addition to the standard gas dynamics. Mock Chandra observations of these simulated clusters are performed by using the true gas density, temperature, and metallicity in each gas cell along with the MEKAL spectral emissivity code (Mewe et al. 1985) to generate an energy-dependent X-ray flux between 0.1 and $10 \mathrm{keV}$. This flux is then projected along the line of sight, converted to a photon count rate appropriate for $z=$ 0.01 , and convolved with an instrumental response simulating that of the Advanced CCD Imaging Spectrometer (ACIS) Imager between 0.7 and $7.0 \mathrm{keV}$. This generates a counts image similar to the Chandra level 2 event files described in $\S 3$. The spectroscopic temperature over a region of interest is determined using an absorbed MEKAL model in XSPEC (Nagai et al. 2006). Temperatures derived using the MEKAL model differ by at most a few percent from those derived from the relativistic Raymond-Smith model used in our analysis. These temperatures should therefore well approximate the measured Chandra temperatures for the clusters in our sample.

We have fit $\beta$-models to the counts images described above for four different simulated clusters, spanning a range in mass from $8.7 \times 10^{13}$ to $6.0 \times 10^{14} M_{\odot}$ at $r_{2500}$ and including both relaxed and unrelaxed clusters. Three fits are performed for each cluster: one each to projections along three perpendicular axes. The likelihood analysis follows the methods of $\S 4.2$, and the central $100 \mathrm{kpc}$ of each simulated cluster is excluded from the fit. Any obvious gas clumps are also removed from the mock images prior to fitting. A spectroscopic temperature is measured between $100 \mathrm{kpc}$ and $r_{2500}$, and the masses are determined at $r_{2500}$. Good- ness values for $100 \mathrm{kpc}$ cut fits to the simulated data are consistently in the range $0.35-0.65$, implying an acceptable description of the data.

Figure 2 shows the mass results for both a highly relaxed and a highly unrelaxed cluster. In both the relaxed and unrelaxed clusters, the gas mass is recovered to better than $10 \%$ between $0.5 r_{2500}$ and $2 r_{2500}$ and to better than $5 \%$ within $r_{2500}$ for the 12 different fits. This shows that the simple isothermal $\beta$-model with central $100 \mathrm{kpc}$ excluded does an excellent job of recovering the true three-dimensional gas mass distribution from the two-dimensional projected data at radii encompassing $r_{2500}$ in simulated clusters.

\section{ADDITIONAL SOURCES OF UNCERTAINTY}

Before presenting the results, we discuss possible sources of both statistical and systematic uncertainty in our measurements and estimate their effects on the X-ray-and SZE-derived gas mass fraction in clusters. Bonamente et al. (2006) address sources of statistical and systematic uncertainty in the X-ray and SZE measurements as they apply to calculation of the angular diameter distance $D_{A}$. Some are found to have a negligible effect on our measurements, such as uncertainties in the assumed Galactic $N_{\mathrm{H}}$ used to calculate spectroscopic temperatures and interference associated with other anisotropies in the CMB. However, we do review nonnegligible effects discussed in Bonamente et al. (2006) in the context of the $f_{\text {gas }}$ calculations, and we address additional uncertainties unique to the $f_{\text {gas }}$ analysis. The effect of these uncertainties on the gas mass fractions is summarized in Table 3. The effects are quantified below in terms of their impact on the gas mass fraction for individual clusters. Note, however, that in mean results for the sample presented in $\S 6.1$, statistical uncertainties average down by a factor of the square root of the sample size, while systematic uncertainties do not average down.

\subsection{Selection Effects}

Clusters chosen for SZE observation are generally selected on the basis of X-ray luminosity, $L_{\mathrm{X}}$. Selecting by X-ray brightness could bias the SZE sample toward elliptical clusters elongated along the line of sight; selecting instead by luminosity alleviates this problem. However, selecting by $L_{\mathrm{X}}$ could introduce other biases because of the preferential selection of cool-core clusters or clusters undergoing major merging events. Another SZE selection bias involves point sources; preferentially selecting clusters without bright ( $~ 220 \mathrm{mJy}$ ) point sources in the field could

TABLE 3

Sources of Uncertainty in $f_{\text {gas }}$ Measurements

\begin{tabular}{|c|c|c|}
\hline Source & $\begin{array}{c}\text { X-Ray } f_{\text {gas }} \\
(\%)\end{array}$ & $\begin{array}{c}\mathrm{SZE} f_{\mathrm{gas}} \\
(\%)\end{array}$ \\
\hline \multicolumn{3}{|c|}{ Statistical Effects $^{\mathrm{a}}$} \\
\hline Kinetic SZE ....................... & $\ldots$ & \pm 4 \\
\hline Radio point sources ...... & $\ldots$ & \pm 4 \\
\hline 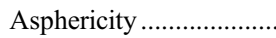 & \pm 20 & \pm 10 \\
\hline \multicolumn{3}{|c|}{ Systematic Effects $^{\mathrm{b}}$} \\
\hline Instrument calibration ... & \pm 6 & \pm 8 \\
\hline X-ray background .......... & +2 & $\ldots$ \\
\hline HSE & -10 & -10 \\
\hline Isothermality ${ }^{\mathrm{c}} \ldots \ldots \ldots . .$. & -5 & -10 \\
\hline
\end{tabular}

\footnotetext{
a Statistical uncertainties average down by a factor of $\sqrt{38}$ for the sample.

b Systematic uncertainties do not average down.

c Applies to isothermal $100 \mathrm{kpc}$ cut model only.
} 
bias our sample toward non-cool-core clusters or toward clusters at higher redshifts whose properties might differ from those of nearby clusters (e.g., Grego et al. 2001; Reese et al. 2002). X-ray selection biases could also be a factor, as we only include clusters that can be fit with the $100 \mathrm{kpc}$ cut and nonisothermal double $\beta$-models. Three clusters, CL J0152.7-1357, A520, and MACS $\mathrm{J} 0717.5+3745$, are not included in our sample because the $\beta$-model provides a poor description of their irregular morphologies when fit to the Chandra data; this is evidenced by the lack of convergence of the $\beta$-model parameters.

We assess the bias associated with sample selection by attempting to construct a subsample largely free of these effects. The subsample consists of clusters from the Brightest Cluster Survey (BCS) catalog (Ebeling et al. 1998), which is compiled with data from a single instrument, Röntgensatellit (ROSAT), and has a well-defined flux limit of $4.4 \times 10^{-12} \mathrm{ergs} \mathrm{cm}^{-2} \mathrm{~s}^{-1}$ in the ROSAT $0.1-2.4 \mathrm{keV}$ band. The subsample contains only clusters with redshifts $z \leq 0.3$ to minimize biases associated with cluster redshift evolution. To reduce selection effects associated with lineof-sight elongation, we impose a flux cut of $6.6 \times 10^{-12} \mathrm{ergs} \mathrm{cm}^{-2}$ $\mathrm{s}^{-1}$ on the subsample, which is $50 \%$ higher than the BCS flux limit. We further select clusters with intrinsic luminosities in the range $0.35 \times 10^{45} \mathrm{ergs} \mathrm{s}^{-1} \leq L_{\mathrm{X}} \leq 1.5 \times 10^{45} \mathrm{ergs} \mathrm{s}^{-1}$ in the 2$10 \mathrm{keV}$ Chandra band. This eliminates clusters in the extreme highand low-luminosity ranges of our sample, which may have unusual dynamical properties. The final subsample consists of the 10 clusters A267, A665, A773, Zw 3146, A1413, A1914, A2204, A2218, A2261, and R2129 (A1413 and R2129 are not included when the SZE-only model is considered, for reasons discussed in $\S 6.1$ ).

To quantify the effects of selection biases, we calculate the weighted mean $f_{\text {gas }}$ values using the $100 \mathrm{kpc}$ cut, nonisothermal, and SZE-only model results (see $\S 6.1$ ) for both the full cluster sample and the unbiased subsample. We find that the mean $f_{\text {gas }}$ changes by less than $3 \%$ between the full and unbiased samples, for all three of these models. We therefore conclude that selection effects do not appear to have a substantial impact on the gas mass fraction or cosmological results. Exclusion of the three aforementioned irregular clusters should also have a small impact on the mean sample results presented below, as they represent just $7 \%$ of the total sample size.

\subsection{Kinetic SZE}

The kinetic SZE is a distortion in the CMB spectrum caused by the peculiar velocity of a cluster along the line of sight. Reese et al. (2002) report that for a cluster with $T_{\mathrm{X}}=8.0 \mathrm{keV}$ and with a typical line-of-sight peculiar velocity of $300 \mathrm{~km} \mathrm{~s}^{-1}$ (Watkins 1997; Colberg et al. 2000), the kinetic SZE would be $4 \%$ of the thermal SZE for our $30 \mathrm{GHz}$ observations. This effect can be positive or negative depending on whether the cluster is moving toward us or away from us. Since the SZE-derived $f_{\text {gas }}$ is linearly proportional to the SZE decrement, the kinetic SZE introduces a $\pm 4 \%$ additional statistical uncertainty into the $f_{\text {gas }}$ measurement of each cluster.

\subsection{Radio Point-Source Contamination}

Point sources unaccounted for in the SZE data can cause the decrement to be "filled in" by the point-source flux, diminishing the magnitude of the SZE signal. It is also possible to overestimate the magnitude of the decrement if the point source is in a negative beam sidelobe. Point sources are identified in the data using images from DIFMAP. All cluster fields are also crossreferenced with $1.4 \mathrm{GHz}$ maps from the NRAO VLA Sky Survey (NVSS; Condon et al. 1998), which occasionally allow us to distinguish faint point sources with flat or gently declining spectra from the noise in the $30 \mathrm{GHz}$ maps. For cases in which NVSS shows a bright source that does not show up at $30 \mathrm{GHz}$, we place a point-source model at the NVSS position and marginalize over its flux. Strongly inverted sources with $30 \mathrm{GHz}$ fluxes near the noise limit would not show up in NVSS but should be rare. Cooray et al. (1998) report spectral indices for 55 point sources with fluxes measured between 1.4 and $30 \mathrm{GHz}$, finding only three with mildly inverted spectra.

NVSS contains only point sources with fluxes greater than $2 \mathrm{mJy}$ at $1.4 \mathrm{GHz}$. To test the effect of point sources too faint to be cross-correlated with NVSS, LaRoque et al. (2002) randomly distribute point sources in the field of the cluster A2163 according to a number count versus flux density relationship calibrated from 41 clusters observed with BIMA. By considering undetected point sources with intrinsic fluxes between 10 and $300 \mu \mathrm{Jy}$ at $30 \mathrm{GHz}$, they find that the best-fit SZE decrement can change by $\pm 4 \%$.

Most clusters have massive central cD galaxies, and many of them are radio bright. LaRoque et al. (2002) include a point-source model at the optical position of the $\mathrm{CD}$ galaxy in A2163 and marginalize over its flux. They find an increase in magnitude of the best-fit central decrement of $2 \%$, and the best-fit point-source flux is consistent with zero. Reese et al. (2002) use a similar treatment, placing point-source models at the centers of the clusters A697, A2261, and A1413. The best-fit fluxes are all consistent with zero and again increase the magnitude of $\Delta T_{0}$ by only $\sim 2 \%$. We conclude that the effect of undetected point sources is likely dominated by the off-center sources and should add an additional $\sim \pm 4 \%$ statistical uncertainty to the SZE gas mass fractions for each cluster.

\subsection{Instrument Calibration}

The absolute calibration of the ACIS response is uncertain at the $5 \%$ level in the $0.7-7.0 \mathrm{keV}$ band, ${ }^{9}$ after CTI corrections and correction for contamination on the optical blocking filters are applied. Measurements of $T_{\mathrm{X}}$ are also subject to $\sim 5 \%$ systematic uncertainty, owing mainly to calibration errors between energy bins. The Chandra calibration uncertainty therefore translates to $\sim 3 \%$ uncertainty in the X-ray gas mass $\left(M_{\text {gas }}^{\mathrm{X}-\text {-ray }} \propto S_{\mathrm{X} 0}^{1 / 2}\right)$ and $5 \%$ in the HSE total mass $\left(M_{\text {total }} \propto T_{\mathrm{X}}\right)$ for a $6 \%$ uncertainty in the $\mathrm{X}$-ray $f_{\text {gas }}$. Absolute calibration of the BIMA/OVRO interferometric imaging system is known conservatively to $4 \%$ (Reese et al. 2002), which when combined with the uncertainty in $T_{\mathrm{X}}$ translates to a $6 \%$ systematic uncertainty in the SZE-derived gas mass $\left(M_{\mathrm{gas}}^{\mathrm{SZE}} \propto \Delta T_{0} / T_{\mathrm{X}}\right)$ and $8 \%$ systematic uncertainty in the SZE-derived $f_{\text {gas }}$.

\subsection{X-Ray Background}

The X-ray background is usually estimated from regions as far from a cluster as possible; however, due to space constraints on the chip, the background regions might contain some amount of cluster flux, particularly for low-z clusters that subtend a larger solid angle on the sky. We therefore test the effect of overestimating the $\mathrm{X}$-ray background due to cluster contamination on a relatively nearby cool-core cluster, Zw 3146. This cluster is on chip I3, so the original background is extracted from circular regions on chips I0, I1, and $\mathrm{I} 2$ at appropriate distances from the readouts (e.g., Bonamente et al. 2004). To assess the effect of overestimating the background, we move the extraction regions closer to the cluster until the best-fit background increases by $\sim 25 \%$ due to cluster contamination. This should be seen as an extreme case, only applicable to a small number of bright, nearby clusters in our sample.

\footnotetext{
9 See http://cxc.harvard.edu/cal/.
} 
The spectral fits are redone with these new background regions, and the resulting temperature and higher background level are applied in the X-ray/SZE joint fit.

We find that the higher X-ray background modifies the bestfit parameters, but the magnitude of the effect on individual parameters is small ( $\lesssim 5 \%$ ) with a combined effect on $f_{\text {gas }}$ of $\sim-7 \%$. We repeat this same analysis on one of the non-cool-core clusters, A2259, which has a much smaller central surface brightness- tobackground ratio, and find that the results are much the same. Given that the typical statistical uncertainty in $B_{\mathrm{X}}$ is at most $\sim 5 \%$, much less than the systematic uncertainty of $25 \%$ considered here, fixing the background in our analysis should have a negligible effect on the $f_{\text {gas }}$ results presented in this paper. To account for the fact that some clusters are more susceptible than others to an overestimated X-ray background, we add a onesided $+2 \%$ systematic uncertainty to the $\mathrm{X}$-ray-derived $f_{\text {gas }}$ results.

\subsection{Effects of Asphericity}

All cluster analysis presented in this paper uses a spherical model. Here we assess the effects of this assumption on the results presented in this paper. To assess this effect, we refit the $\mathrm{X}$-ray data of all 38 clusters using a $100 \mathrm{kpc}$ cut isothermal $\beta$-model and accounting for projected ellipticity $\eta$ and position angle of the clusters on the sky. We find a mean projected ellipticity $\langle\eta\rangle=0.79$ with rms scatter 0.09 , where $\eta$ is the ratio of a cluster's minor axis to its major axis. These results are consistent with the previous studies of Mohr et al. (1995). If the inclination angle is accounted for (e.g., Cooray 1998), an ellipsoidal cluster with $\eta=0.8$ and an axis of symmetry inclined at $45^{\circ}$ to the line of sight can have a derived total mass in error by $12 \%$ and a derived gas mass in error by $6 \%$ if it is modeled as a sphere. We extend this to the more complex triaxial distributions and conservatively estimate a statistical uncertainty of $10 \%$ in the X-rayderived $M_{\text {gas }}$ and $15 \%$ in $M_{\text {total }}$. This translates to a $\sim 20 \%$ statistical uncertainty in the X-ray-derived gas mass fractions, given that the changes in gas mass and total mass can either add or partially cancel depending on inclination angle and geometry (Piffaretti et al. 2003). The effect on the SZE $f_{\text {gas }}$ results should be smaller, since the SZE depends linearly on density. Grego et al. (2000) calculate the gas mass fraction of A370 assuming both prolate and oblate geometries and find a resulting uncertainty in $f_{\text {gas }}$ of $20 \%$. This cluster has $\eta=0.64$, however, so we assume a milder $10 \%$ statistical uncertainty in the $\mathrm{SZE} f_{\text {gas }}$ results for each cluster.

\subsection{Hydrostatic Equilibrium}

The HSE condition is a key assumption that enables measurements of the gravitationally bound mass of clusters from the $\mathrm{X}$-ray and SZE data. The assumption is that the ICM is supported against gravitational collapse only by thermal pressure of the hot intracluster gas. The hydrostatic mass would therefore underestimate the true gravitationally bound mass of the cluster if nonthermal pressure support is present. One form of nonthermal pressure comes from turbulent gas motions in clusters. Recent numerical simulations suggest that this provides about $10 \%$ of the total pressure support in clusters (Faltenbacher et al. 2005; Rasia et al. 2006; E. Lau et al. 2006, in preparation), and similar results are obtained using XMM-Newton observations of the Coma Cluster (Schuecker et al. 2004). More direct measurements of the turbulent gas motions in clusters, however, await measurements of Doppler broadening of the iron lines with high-resolution X-ray spectroscopy experiments (Inogamov \& Sunyaev 2003). Cosmic rays and magnetic fields may also provide nonthermal pressure support. While direct measurements of the nonthermal pressure support may be difficult, detailed comparisons of the hydrostatic mass with other mass estimates for the same cluster (e.g., gravitational lensing and the velocity dispersion of galaxies in clusters) will likely provide an important check on the hydrostatic mass estimate and potentially interesting constraints on the nonthermal pressure support in clusters. For now we assign a onesided systematic uncertainty of $-10 \%$ to the total masses, which accounts for the assumed contribution from nonthermal pressure. We caution, however, that this estimate is somewhat uncertain and that this effect will need to be investigated in greater detail as more data become available.

\subsection{Isothermal Assumption}

The isothermal assumption can potentially affect the gas mass fraction measurements and the cosmological constraints through its effects on both gas mass and total mass. The X-ray-derived gas mass is affected little by the isothermal assumption because the X-ray emission has a very weak dependence on the assumed temperature $\left(\Lambda \propto T^{1 / 2}\right)$. The SZE-derived gas mass is more sensitive to this assumption, since it depends linearly on the cluster gas temperature. Our choice of a radial range between $100 \mathrm{kpc}$ and $r_{2500}$ should minimize the bias due to the temperature structure for two reasons: (1) use of the X-ray temperature with the $100 \mathrm{kpc}$ core exclusion should avoid the most significant bias associated with cool-core clusters, and (2) focusing on the inner $r_{2500}$ should minimize the bias due to the temperature decline observed in the outskirts of clusters (Vikhlinin 2006).

The total mass derived from the X-ray and SZE data is also affected by the isothermal assumption. If the temperature profile deviates from the isothermal gas distribution, the average temperature depends on the weighting scheme. For example, the spectroscopic temperature, $T_{\text {spec }}$, may differ by about $10 \%$ from the gas mass-weighted temperature $T_{\mathrm{mg}}$ in the radial range between $70 \mathrm{kpc}$ and $r_{500}$ (Vikhlinin 2006). But the difference is likely smaller in the radial range $\left(100 \mathrm{kpc}<r<r_{2500}\right)$ considered in this study. We therefore expect that the bias on the cluster masses, and hence on our gas mass fraction measurements, is not significant.

The effect of the isothermal assumption can be quantified using the results of Table 7, presented in $\S 6.1$ below. There we find that the X-ray- and SZE-weighted mean gas mass fractions are nearly identical for the nonisothermal model, while the SZE gas mass fraction is $\sim 5 \%$ larger than the X-ray value for the $100 \mathrm{kpc}$ cut model. This hints at a possible systematic bias of about $-5 \%$ in the spectral temperature under the isothermal assumption, since the SZE $f_{\text {gas }}$ has an additional $T_{\mathrm{X}}^{-1}$ dependence relative to the $\mathrm{X}$-ray value. We therefore assign an additional $-5 \%$ systematic uncertainty to the gas mass fractions derived from the X-ray $100 \mathrm{kpc}$ cut fits and a $-10 \%$ systematic uncertainty to the gas mass fractions from the SZE $100 \mathrm{kpc}$ cut fits.

\subsection{Cluster Substructure}

The X-ray-derived gas mass fractions are particularly sensitive to any nonnegligible small-scale structure in the intracluster gas. When gas clumps, it has a density higher than the local average, $\left\langle n_{e}\right\rangle$, and the X-ray surface brightness is increased by a factor of $C \equiv\left\langle n_{e}^{2}\right\rangle /\left\langle n_{e}\right\rangle^{2}$ relative to the SZE decrement (Nagai et al. 2000; Mathiesen \& Evrard 2001). Since $n_{e} \propto S_{\mathrm{X}}^{1 / 2}$ for the X-ray data and $n_{e} \propto \Delta T$ for the SZE data, X-ray gas mass fractions would be systematically overestimated by a factor of $C^{1 / 2}$ with respect to SZE gas mass fractions in the presence of significant clumping. Cluster substructure has two important effects on the $\mathrm{X}$-ray observables. First, gas clumps can bias the measured X-ray 
TABLE 4

Model Parameters, Cluster Masses, and Gas Mass Fractions: Isothermal $\beta$-Model with 100 kpc Cut

\begin{tabular}{|c|c|c|c|c|c|c|c|c|c|c|c|}
\hline Cluster & $S_{\mathrm{X} 0}{ }^{\mathrm{a}}$ & $\begin{array}{c}\theta_{c} \\
(\operatorname{arcsec})\end{array}$ & $\beta$ & $\begin{array}{l}\Delta T_{0} \\
(\mathrm{mK})\end{array}$ & $\begin{array}{c}T_{\mathrm{X}} \\
(\mathrm{keV})\end{array}$ & $\begin{array}{c}M_{\text {gas }}^{X} \\
\left(10^{13} M_{\odot}\right)\end{array}$ & $\begin{array}{c}M_{\text {gas }}^{\text {SZE }} \\
\left(10^{13} M_{\odot}\right)\end{array}$ & $\begin{array}{c}M_{\text {total }} \\
\left(10^{14} M_{\odot}\right)\end{array}$ & $f_{\text {gas }}^{\mathrm{X} \text {-ray }}$ & $f_{\mathrm{gas}}^{\mathrm{SZE}}$ & $t_{\text {cool }} / t_{\text {Hubble }}$ \\
\hline CL $0016 \ldots \ldots \ldots \ldots \ldots$ & $1.27 \pm 0.04$ & $42.8_{-2.0}^{+2.1}$ & $0.742_{-0.022}^{+0.025}$ & $-1.361 \pm 0.083$ & $10.46_{-0.60}^{+0.61}$ & $4.38 \pm 0.29$ & $4.34 \pm 0.26$ & $3.33_{-0.37}^{+0.39}$ & $0.131_{-0.006}^{+0.007}$ & $0.130_{-0.015}^{+0.016}$ & 1.5 \\
\hline A68........................ & $1.60_{-0.11}^{+0.13}$ & $55.1_{-5.0}^{+5.3}$ & $0.764_{-0.042}^{+0.046}$ & $-0.712_{-0.103}^{+0.099}$ & $9.55_{-0.96}^{+1.09}$ & $3.65_{-0.31}^{+0.34}$ & $3.07_{-0.44}^{+0.45}$ & $4.32_{-0.74}^{+0.91}$ & $0.084_{-0.008}^{+0.009}$ & $0.071_{-0.016}^{+0.019}$ & 1.4 \\
\hline A267...................... & $2.04_{-0.23}^{+0.28}$ & $43.1_{-5.1}^{+5.8}$ & $0.720_{-0.040}^{+0.049}$ & $-0.692_{-0.080}^{+0.077}$ & $5.89_{-0.54}^{+0.66}$ & $2.24_{-0.18}^{+0.20}$ & $2.83_{-0.31}^{+0.32}$ & $2.03_{-0.33}^{+0.44}$ & $0.110 \pm 0.011$ & $0.140_{-0.029}^{+0.032}$ & 0.8 \\
\hline A370 ....................... & $0.73 \pm 0.02$ & $61.8_{-3.5}^{+3.6}$ & $0.811_{-0.037}^{+0.039}$ & $-0.866_{-0.088}^{+0.090}$ & $8.67_{-0.49}^{+0.51}$ & $2.78_{-0.20}^{+0.21}$ & $3.51_{-0.36}^{+0.37}$ & $2.77_{-0.33}^{+0.37}$ & $0.100 \pm 0.005$ & $0.127_{-0.018}^{+0.020}$ & 2.1 \\
\hline MS $0451 \ldots \ldots \ldots \ldots$ & $2.35_{-0.10}^{+0.11}$ & $40.0_{-1.7}^{+1.9}$ & $0.773_{-0.023}^{+0.026}$ & $-1.476 \pm 0.086$ & $9.95_{-0.69}^{+0.76}$ & $4.76_{-0.30}^{+0.31}$ & $4.79 \pm 0.28$ & $3.76_{-0.46}^{+0.53}$ & $0.127_{-0.008}^{+0.009}$ & $0.127_{-0.018}^{+0.020}$ & 1.0 \\
\hline MC $0647 \ldots \ldots \ldots \ldots . . . .$. & $2.19_{-0.18}^{+0.20}$ & $24.1_{-1.8}^{+2.0}$ & $0.687_{-0.020}^{+0.023}$ & $-1.312_{-0.123}^{+0.116}$ & $14.06_{-1.59}^{+1.78}$ & $4.90_{-0.42}^{+0.47}$ & $3.26_{-0.29}^{+0.30}$ & $5.98_{-1.05}^{+1.30}$ & $0.082_{-0.008}^{+0.009}$ & $0.055_{-0.012}^{+0.014}$ & 1.0 \\
\hline A586........................ & $3.36_{-0.36}^{+0.43}$ & $45.3_{-4.6}^{+5.1}$ & $0.723_{-0.033}^{+0.039}$ & $-0.651_{-0.084}^{+0.081}$ & $6.35_{-0.39}^{+0.46}$ & $2.26_{-0.11}^{+0.13}$ & $2.49 \pm 0.32$ & $2.48_{-0.28}^{+0.34}$ & $0.091 \pm 0.007$ & $0.100_{-0.017}^{+0.012}$ & 0.5 \\
\hline 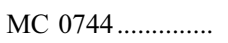 & $1.40_{-0.10}^{+0.12}$ & $25.8 \pm 2.0$ & $0.723_{-0.027}^{+0.023}$ & $-1.299_{-0.138}^{-0.0134}$ & $8.14_{-0.72}^{+0.89}$ & $3.07_{-0.25}^{+0.21}$ & $3.36_{-0.35}^{+0.34}$ & $2.26_{-0.35}^{+0.48}$ & $0.136_{-0.011}^{+0.012}$ & $0.148_{-0.027}^{+0.031}$ & 0.3 \\
\hline A611 ....................... & $3.21_{-0.29}^{+0.37}$ & $25.7_{-2.2}^{+2.1}$ & $0.632 \pm 0.017$ & $-0.761_{-0.082}^{+0.084}$ & $6.79_{-0.38}^{+0.41}$ & $2.36 \pm 0.11$ & $2.33 \pm 0.26$ & $2.14_{-0.20}^{+0.22}$ & $0.110 \pm 0.006$ & $0.109_{-0.016}^{+0.017}$ & 0.6 \\
\hline A665 ........................ & $6.51_{-1.11}^{+1.46}$ & $17.7_{-2.5}^{+2.9}$ & $0.454_{-0.005}^{+0.006}$ & $-1.330_{-0.119}^{+0.117}$ & $8.37_{-0.33}^{+0.37}$ & $2.62_{-0.09}^{+0.10}$ & $2.53_{-0.23}^{+0.22}$ & $2.00_{-0.12}^{+0.10}$ & $0.131 \pm 0.004$ & $0.126_{-0.013}^{+0.010}$ & 1.1 \\
\hline A697........................ & $2.46 \pm 0.06$ & $42.7_{-1.5}^{+1.6}$ & $0.605 \pm 0.011$ & $-1.223_{-0.121}^{+0.1125}$ & $10.21_{-0.65}^{+0.70}$ & $4.39_{-0.26}^{+0.28}$ & $4.09_{-0.42}^{+0.41}$ & $3.47_{-0.37}^{+0.40}$ & $0.127_{-0.006}^{+0.007}$ & $0.117_{-0.017}^{+0.019}$ & 1.2 \\
\hline A773 ....................... & $2.31_{-0.15}^{+0.17}$ & $38.7 \pm 2.5$ & $0.594_{-0.012}^{+0.013}$ & $-1.101_{-0.116}^{+0.118}$ & $8.16_{-0.52}^{+0.56}$ & $2.75_{-0.15}^{+0.17}$ & $3.64 \pm 0.39$ & $2.59_{-0.27}^{+0.30}$ & $0.106_{-0.005}^{+0.006}$ & $0.140_{-0.021}^{+0.024}$ & 1.0 \\
\hline 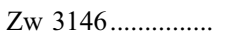 & $7.56_{-0.28}^{+0.32}$ & $30.4 \pm 0.9$ & $0.736 \pm 0.009$ & $-1.172_{-0.144}^{+0.143}$ & $8.28_{-0.29}^{+0.30}$ & $4.42 \pm 0.11$ & $3.80 \pm 0.47$ & $3.62_{-0.20}^{+0.22}$ & $0.122 \pm 0.004$ & $0.105_{-0.014}^{+0.015}$ & 0.1 \\
\hline MS $1054 \ldots \ldots \ldots \ldots . . . . .$. & $0.43 \pm 0.02$ & $57.7_{-7.5}^{+8.8}$ & $0.884_{-0.117}^{+0.155}$ & $-1.183_{-0.135}^{+0.125}$ & $9.77_{-0.94}^{+1.10}$ & $1.06_{-0.49}^{+0.50}$ & $1.12_{-0.47}^{+0.42}$ & $0.74_{-0.38}^{+0.45}$ & $0.144_{-0.013}^{+0.014}$ & $0.153_{-0.029}^{+0.034}$ & 2.8 \\
\hline 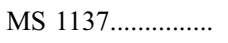 & $0.62_{-0.08}^{+0.11}$ & $22.3_{-3.4}^{+3.8}$ & $0.877_{-0.078}^{+0.097}$ & $-0.775_{-0.102}^{+0.093}$ & $4.47_{-0.44}^{+0.53}$ & $1.19_{-0.13}^{+0.14}$ & $2.13_{-0.25}^{+0.26}$ & $1.03_{-0.20}^{+0.26}$ & $0.115_{-0013}^{+0.014}$ & $0.207_{-0.045}^{+0.054}$ & 0.7 \\
\hline 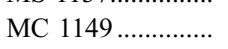 & $0.81 \pm 0.04$ & $44.7_{-2.3}^{+2.6}$ & $0.666_{-0.018}^{+0.020}$ & $-1.166_{-0.114}^{+0.102}$ & $9.93_{-0.68}^{+0.84}$ & $3.09_{-0.30}^{-0.13}$ & $3.00 \pm 0.30$ & $2.31_{-0.34}^{+0.40}$ & $0.134 \pm 0.008$ & $0.130_{-0.019}^{+0.045}$ & 1.9 \\
\hline 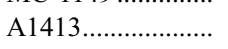 & $4.31_{-0.41}^{+0.59}$ & $37.9_{-4.3}^{+3.3}$ & $0.536 \pm 0.015$ & $-1.037 \pm 0.150$ & $7.51_{-0.29}^{+0.35}$ & $2.63_{-0.10}^{+0.110}$ & $2.98_{-0.42}^{+0.43}$ & $2.15_{-0.15}^{+0.17}$ & $0.122 \pm 0.004$ & $0.139 \pm 0.022$ & 0.3 \\
\hline 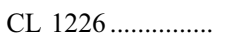 & $2.36_{-0.48}^{+0.71}$ & $16.2_{-3.3}^{+3.8}$ & $0.732_{-0.060}^{+0.084}$ & $-1.715_{-0.234}^{+0.202}$ & $13.47_{-2.20}^{+2.72}$ & $3.89_{-0.46}^{+0.51}$ & $2.90_{-0.30}^{+0.34}$ & $5.21_{-1.39}^{+1.96}$ & $0.075_{-0.014}^{+0.015}$ & $0.056_{-0.018}^{+0.024}$ & 0.9 \\
\hline MC $1311 \ldots \ldots \ldots \ldots$ & $1.03_{-0.46}^{+0.58}$ & $7.8_{-1.5}^{+2.9}$ & $0.621_{-0.022}^{+0.030}$ & $-1.489_{-0.314}^{+0.309}$ & $7.16_{-1.15}^{+1.53}$ & $2.11_{-0.21}^{+0.24}$ & $2.33 \pm 0.38$ & $2.17_{-0.53}^{+0.74}$ & $0.097_{-0.017}^{+0.019}$ & $0.107_{-0.035}^{+0.047}$ & 0.3 \\
\hline 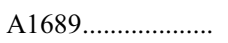 & $6.26_{-0.26}^{+0.40}$ & $48.4_{-2.0}^{+2.1}$ & $0.688_{-0.012}^{+0.013}$ & $-1.651_{-0.137}^{-0.130}$ & $10.46_{-0.47}^{+0.46}$ & $5.06_{-0.17}^{+0.16}$ & $5.85_{-0.47}^{+0.49}$ & $4.96 \pm 0.36$ & $0.102 \pm 0.004$ & $0.118_{-0.013}^{+0.013}$ & 0.3 \\
\hline RX J1347 ................ & $15.6_{-0.60}^{+0.70}$ & $17.7_{-0.8}^{+0.9}$ & $0.651 \pm 0.007$ & $-2.777_{-0.284}^{+0.294}$ & $16.48_{-0.92}^{+0.99}$ & $8.84_{-0.35}^{+0.37}$ & $5.60_{-0.60}^{+0.58}$ & $8.08_{-0.69}^{+0.77}$ & $0.109_{-0.005}^{+0.006}$ & $0.069_{-0.010}^{+0.011}$ & 0.1 \\
\hline MS $1358 \ldots \ldots \ldots \ldots \ldots$ & $1.79_{-0.11}^{+0.13}$ & $28.9_{-1.9}^{+1.8}$ & $0.638 \pm 0.016$ & $-0.721_{-0.097}^{+0.096}$ & $8.93_{-0.74}^{+0.88}$ & $2.53_{-0.17}^{+0.19}$ & $2.23 \pm 0.29$ & $3.13_{-0.41}^{+0.51}$ & $0.081 \pm 0.006$ & $0.071_{-0.014}^{+0.010}$ & 0.2 \\
\hline A1835 & $9.49_{-0.32}^{+0.36}$ & $33.6 \pm 1.0$ & $0.690_{-0.008}^{+0.007}$ & $-1.636_{-0.114}^{+0.116}$ & $11.44_{-0.56}^{+0.66}$ & $5.78_{-0.20}^{+0.23}$ & $4.98 \pm 0.36$ & $5.56_{-0.42}^{+0.50}$ & $0.104 \pm 0.005$ & $0.089_{-0.010}^{+0.011}$ & 0.1 \\
\hline 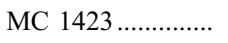 & $3.21_{-0.34}^{+0.39}$ & $14.6_{-1.0}^{+1.2}$ & $0.637_{-0.011}^{+0.010}$ & $-1.177_{-0.200}^{+0.210}$ & $6.97_{-0.40}^{+0.42}$ & $2.26 \pm 0.10$ & $2.63_{-0.47}^{+0.44}$ & $1.94_{-0.18}^{+0.20}$ & $0.116 \pm 0.006$ & $0.135_{-0.028}^{+0.029}$ & 0.1 \\
\hline A1914.................... & $10.3 \pm 0.40$ & $44.0 \pm 1.3$ & $0.734_{-0.010}^{+0.009}$ & $-1.565 \pm 0.125$ & $9.48_{-0.29}^{+0.35}$ & $4.85_{-0.10}^{+0.12}$ & $5.11 \pm 0.42$ & $4.82_{-0.25}^{+0.30}$ & $0.100_{-0.004}^{+0.003}$ & $0.106 \pm 0.011$ & 0.7 \\
\hline A1995 & $1.62 \pm 0.05$ & $57.5_{-2.6}^{+2.7}$ & $1.013_{-0.038}^{+0.041}$ & $-0.879_{-0.052}^{+0.053}$ & $8.22_{-0.45}^{+0.44}$ & $3.51 \pm 0.14$ & $4.33_{-0.27}^{+0.28}$ & $4.74_{-0.48}^{+0.50}$ & $0.074 \pm 0.005$ & $0.092_{-0.011}^{+0.013}$ & 1.2 \\
\hline 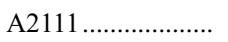 & $0.89_{-0.08}^{+0.10}$ & $51.0_{-6.6}^{+7.6}$ & $0.611_{-0.036}^{+0.038}$ & $-0.596_{-0.122}^{-0.0117}$ & $8.15_{-0.83}^{+0.98}$ & $2.19_{-0.23}^{+0.27}$ & $2.15 \pm 0.42$ & $2.49_{-0.44}^{+0.56}$ & $0.088 \pm 0.008$ & $0.085_{-0.021}^{+0.026}$ & 1.7 \\
\hline 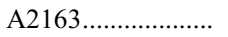 & $4.63 \pm 0.09$ & $57.6_{-1.2}^{+1.3}$ & $0.538 \pm 0.005$ & $-1.903_{-0.1177}^{+0.171}$ & $14.81_{-0.38}^{+0.39}$ & $8.08_{-0.20}^{+0.21}$ & $5.89_{-0.53}^{+0.55}$ & $5.49_{-0.23}^{+0.24}$ & $0.147 \pm 0.003$ & $0.107 \pm 0.011$ & 1.2 \\
\hline A2204..................... & $1.37_{-0.22}^{+0.35}$ & $33.7_{-5.7}^{+6.6}$ & $0.614_{-0.034}^{+0.047}$ & $-1.644 \pm 0.166$ & $11.23_{-0.72}^{+0.80}$ & $4.73_{-0.24}^{+0.20}$ & $4.30_{-0.39}^{+0.37}$ & $4.96_{-0.64}^{+0.80}$ & $0.096_{-0.010}^{+0.009}$ & $0.087 \pm 0.014$ & 0.1 \\
\hline 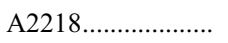 & $1.69_{-0.02}^{+0.03}$ & $70.3 \pm 1.7$ & $0.765_{-0.013}^{+0.014}$ & $-0.870_{-0.078}^{+0.079}$ & $7.80_{-0.37}^{+0.41}$ & $3.00_{-0.12}^{+0.13}$ & $4.10 \pm 0.38$ & $3.33_{-0.27}^{+0.34}$ & $0.090 \pm 0.004$ & $0.123_{-0.015}^{+0.017}$ & 1.2 \\
\hline RX J1716 .................. & $0.55_{-0.13}^{+0.22}$ & $17.6_{-4.5}^{+5.7}$ & $0.665_{-0.064}^{+0.097}$ & $-0.656_{-0.166}^{+0.148}$ & $6.57_{-0.88}^{+1.08}$ & $1.23_{-0.16}^{+0.19}$ & $1.32 \pm 0.28$ & $1.39_{-0.33}^{+0.45}$ & $0.088_{-0.011}^{+0.013}$ & $0.093_{-0.028}^{+0.034}$ & 1.0 \\
\hline A2259.................... & $1.48_{-0.14}^{+0.19}$ & $59.1_{-8.4}^{+9.1}$ & $0.663_{-0.048}^{+0.057}$ & $-0.397_{-0.139}^{+0.138}$ & $5.81_{-036}^{+0.44}$ & $1.81 \pm 0.14$ & $1.65_{-0.58}^{+0.61}$ & $1.79_{-0.24}^{+0.27}$ & $0.101 \pm 0.007$ & $0.092_{-0.034}^{+0.036}$ & 0.8 \\
\hline A2261.................... & $5.92_{-0.77}^{+0.96}$ & $28.9_{-3.0}^{+3.5}$ & $0.624_{-0.018}^{+0.023}$ & $-1.178_{-0.121}^{+0.1122}$ & $7.45_{-0.55}^{+0.65}$ & $3.04_{-0.19}^{+0.20}$ & $3.58_{-0.38}^{+0.48}$ & $2.56_{-0.31}^{+0.36}$ & $0.119 \pm 0.008$ & $0.139_{-0.023}^{+0.026}$ & 0.3 \\
\hline MS $2053 \ldots \ldots \ldots \ldots . . . . .$. & $0.29_{-0.05}^{+0.06}$ & $27.0_{-4.8}^{+6.0}$ & $0.821_{-0.085}^{+0.1122}$ & $-0.419_{-0.078}^{+0.069}$ & $4.77_{-0.58}^{+0.71}$ & $0.93 \pm 0.12$ & $1.39_{-0.22}^{+0.20}$ & $1.23_{-0.29}^{+0.31}$ & $0.076_{-0.011}^{+0.012}$ & $0.113_{-0.030}^{+0.039}$ & 0.9 \\
\hline MC $2129 \ldots \ldots \ldots . .$. & $1.99_{-0.20}^{+0.05}$ & $24.4_{-2.6}^{+2.8}$ & $0.712_{-0.033}^{+0.040}$ & $-1.176_{-0.133}^{+0.0129}$ & $8.55_{-0.80}^{+0.97}$ & $3.26_{-0.26}^{+0.30}$ & $3.28_{-0.35}^{+0.34}$ & $2.82_{-0.45}^{+0.59}$ & $0.116 \pm 0.011$ & $0.116_{-0.023}^{+0.027}$ & 0.8 \\
\hline RX J2129 .................. & $4.99_{-0.83}^{+1.10}$ & $25.4_{-3.3}^{+3.8}$ & $0.611_{-0.020}^{+0.023}$ & $-0.734_{-0.120}^{-0.1133}$ & $6.69_{-0.47}^{+0.40}$ & $2.58_{-0.14}^{+0.16}$ & $2.17 \pm 0.33$ & $2.08_{-0.23}^{+0.27}$ & $0.124 \pm 0.008$ & $0.104_{-0.019}^{+0.023}$ & 0.1 \\
\hline 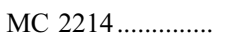 & $1.76_{-0.14}^{+0.15}$ & $31.5_{-2.8}^{+3.1}$ & $0.710_{-0.034}^{+0.038}$ & $-1.433_{-0.126}^{+0.119}$ & $10.22_{-0.88}^{+0.99}$ & $3.95_{-0.32}^{+0.34}$ & $4.50 \pm 0.36$ & $3.85_{-0.59}^{+0.68}$ & $0.103_{-0.008}^{+0.009}$ & $0.117_{-0.020}^{+0.029}$ & 0.9 \\
\hline MC $2228 \ldots \ldots \ldots \ldots . . . .$. & $2.36_{-0.13}^{+0.17}$ & $20.2_{-2.5}^{+2.97}$ & $0.522_{-0.013}^{+0.015}$ & $-1.727_{-0.177}^{+0.166}$ & $8.43_{-0.71}^{+0.78}$ & $2.81_{-0.22}^{+0.24}$ & $3.84_{-0.36}^{+0.35}$ & $2.03_{-0.27}^{+0.31}$ & $0.138 \pm 0.009$ & $0.188_{-0.031}^{+0.035}$ & 0.9 \\
\hline
\end{tabular}

${ }^{a}$ Units are $10^{-12} \mathrm{ergs} \mathrm{cm}^{-2} \operatorname{arcmin}^{-2} \mathrm{~s}^{-1}$ 

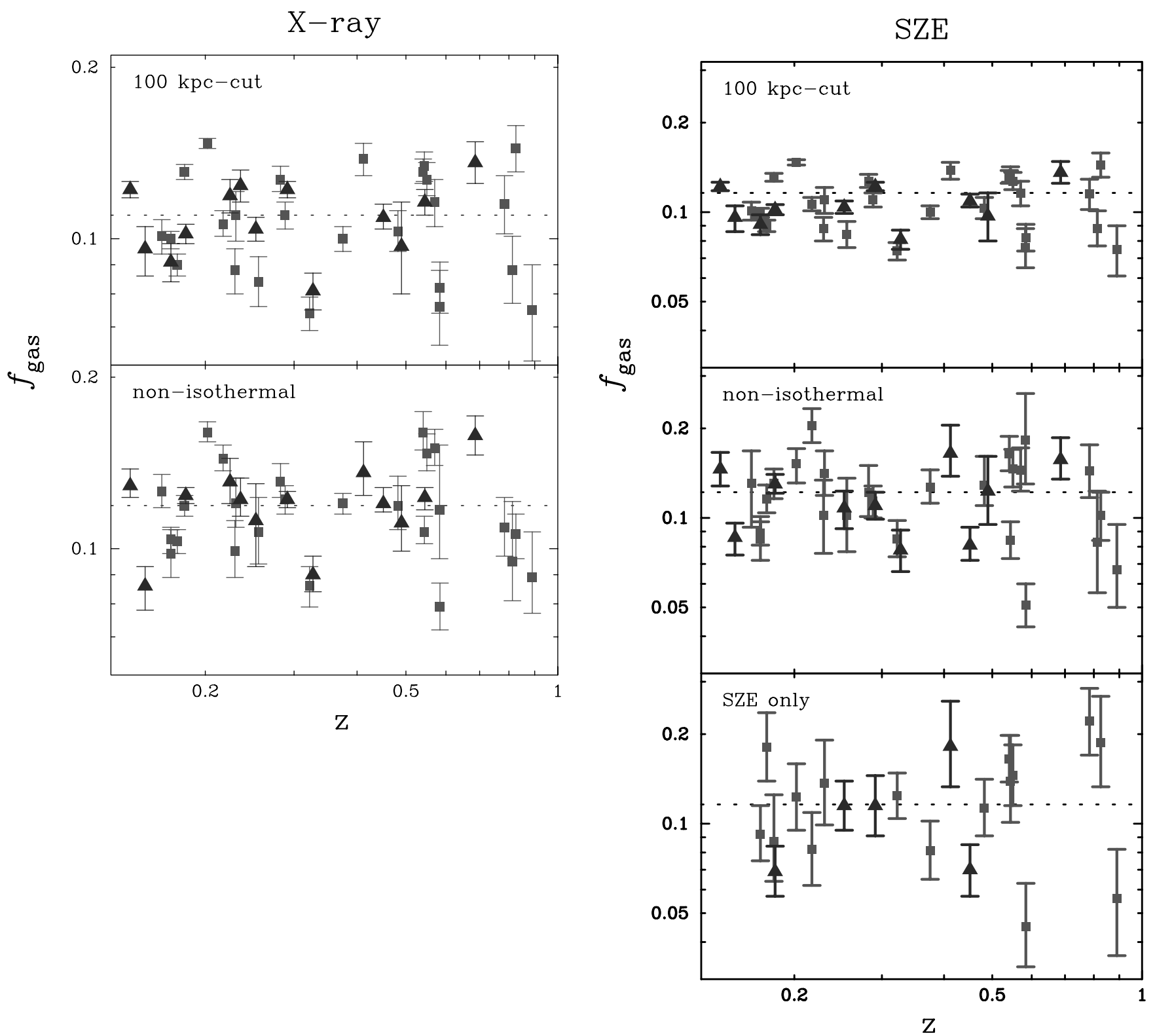

FIG. 3.-Gas mass fraction vs. redshift derived from the X-ray data (left) and from the SZE data (right). Triangles denote cool-core clusters, and squares denote non-cool-core clusters. Dashed lines show the weighted mean $f_{\text {gas }}$ for each sample. Error bars are statistical from Tables 4, 5, and 6. [See the electronic edition of the Journal for a color version of this figure.]

gas mass high by $C^{1 / 2}$. Second, cluster substructure can bias the average spectroscopic temperature of the cluster as a whole (e.g., Mathiesen \& Evrard 2001; Mazzotta et al. 2004).

The Chandra observations provide important handles on both of these effects because their superb resolution and sensitivity enable detection of the prominent clumps that contribute significantly to the X-ray surface brightness and the determination of the average temperature of the cluster. We test this using the mock Chandra observations of the simulated clusters introduced in $\S 4.4$. The test shows that the gas mass is recovered to better than $5 \%$ using the simple $100 \mathrm{kpc}$ cut model, indicating that the clumping factor should be small if the most prominent clump is removed from the image. These results should also hold for the high- $z$ clusters, as a significant fraction of the low-energy photons from the cool clumps are redshifted out of the $0.7-7 \mathrm{keV}$ band, making the instrument less sensitive to clumping effects (Nagai et al. 2006).

For the same reasons, the bias on the Chandra spectroscopic temperature is also small. Tests on several clusters in our sample show that not removing bright clumps could bias the temperatures low by $10 \%-15 \%$, in reasonable agreement with the previous findings (Mathiesen \& Evrard 2001; Mazzotta et al. 2004). Further tests, leaving in only fainter clumps, show a decrease in spectroscopic temperature of only $2 \%-3 \%$, smaller than the assumed bias due to the isothermal assumption.

We therefore conclude that there is no evidence that cluster substructure significantly affects our results when bright spots are removed from the X-ray images prior to fitting. The agreement in the mean X-ray and SZE gas mass fractions, particularly for the nonisothermal model, which avoids the bias due to the isothermal assumption, is consistent with this conclusion (see $\S 6.3$ ).

\section{RESULTS AND DISCUSSION}

\subsection{Gas Mass Fraction Results}

In Table 4 we present the X-ray and SZE measurements of cluster gas mass fractions as determined from the $100 \mathrm{kpc}$ cut 
TABLE 5

Model Parameters, Cluster Masses, and Gas Mass Fractions: Nonisothermal Double $\beta$-Model

\begin{tabular}{|c|c|c|c|c|c|c|c|c|c|c|c|c|c|}
\hline Cluster & $\begin{array}{c}r_{200} \\
(\mathrm{Mpc})\end{array}$ & Concentration & $\begin{array}{c}n_{e 0}^{\mathrm{X} \text {-ray }} \\
\left(10^{-2} \mathrm{~cm}^{-3}\right)\end{array}$ & $\begin{array}{c}n_{e 0}^{\mathrm{SZE}} \\
\left(10^{-2} \mathrm{~cm}^{-3}\right)\end{array}$ & $\begin{array}{c}\theta_{c 1} \\
(\operatorname{arcsec})\end{array}$ & $\beta$ & $f$ & $\begin{array}{c}\theta_{c 2} \\
(\operatorname{arcsec})\end{array}$ & $\begin{array}{c}M_{\text {gas }}^{\mathrm{X} \text {-ray }} \\
\left(10^{13} M_{\odot}\right)\end{array}$ & $\begin{array}{c}M_{\mathrm{gas}}^{\mathrm{SZE}} \\
\left(10^{13} M_{\odot}\right)\end{array}$ & $\begin{array}{c}M_{\text {total }} \\
\left(10^{14} M_{\odot}\right)\end{array}$ & $f_{\text {gas }}^{\mathrm{X} \text {-ray }}$ & $f_{\mathrm{gas}}^{\mathrm{SZE}}$ \\
\hline L $0016 \ldots \ldots \ldots \ldots \ldots$ & $2.12_{-0.22}^{+0.24}$ & & $1.39_{-0.12}^{+0.16}$ & $1.44_{-0.18}^{+0.20}$ & $11.0_{-2.5}^{+3.2}$ & $0.759_{-0.024}^{+0.034}$ & $0.483_{-0.054}^{+0.046}$ & $48.1_{-2.6}^{+3.8}$ & $4.63_{-0.56}^{+0.48}$ & $4.76_{-0.36}^{+0.32}$ & $2.89_{-0.55}^{+0.52}$ & $0.160_{-0.011}^{+0.014}$ & $0.164_{-0.020}^{+0.024}$ \\
\hline A68................. & $22_{-0.32}^{+0.60}$ & & $0.82 \pm 0.03$ & $0.77_{-0.15}^{+0.18}$ & $\ldots$ & $0.707 \pm 0.031$ & & $48.7 \pm 3.3$ & $3.84 \pm 0.50$ & $3.63_{-0.55}^{+0.36}$ & $\begin{array}{l}2.57_{-0.87}^{+0.55} \\
3.98\end{array}$ & $0.107_{-0.013}^{+0.011}$ & $0.102_{-0.025}^{+0.020}$ \\
\hline A267... & $1.55_{-0.20}^{+0.25}$ & $4.19_{-1.48}^{+2.41}$ & $1.04 \pm 0.04$ & $1.22_{-0.16}^{+0.18}$ & $\ldots$ & $0.694 \pm 0.029$ & $\ldots$ & $40.6_{-2.7}^{+2.8}$ & $2.35 \pm 0.19$ & $2.76_{-0.34}^{+0.35}$ & $1.95_{-0.32}^{+0.36}$ & $0.120_{-0.011}^{+0.013}$ & $0.141_{-0.022}^{+0.027}$ \\
\hline A370..................... & $1.51 \pm 0.10$ & $5.32_{-1.40}^{+1.98}$ & $0.54 \pm 0.01$ & $0.57_{-0.06}^{+0.07}$ & $\ldots$ & $0.737_{-0.027}^{+0.031}$ & & $55.5_{-2.4}^{+2.8}$ & $3.02 \pm 0.19$ & $3.20_{-0.31}^{+0.30}$ & $2.52 \pm 0.26$ & $0.120 \pm 0.005$ & $0.127_{-0.015}^{+0.018}$ \\
\hline MS $0451 \ldots \ldots \ldots \ldots$ & $1.97_{-0.33}^{+0.53}$ & $2.18_{-1.07}^{+1.66}$ & $1.35 \pm 0.03$ & $1.33_{-0.14}^{+0.17}$ & $\ldots$ & $0.774_{-0.019}^{+0.021}$ & $\ldots$ & $34.0 \pm 1.2$ & $5.25_{-0.40}^{+0.37}$ & $5.20_{-0.49}^{+0.43}$ & $3.56_{-0.51}^{+0.54}$ & $0.147_{-0.010}^{+0.012}$ & $0.146_{-0.019}^{+0.024}$ \\
\hline MC $0647 \ldots \ldots \ldots \ldots . . .$. & $2.02_{-0.22}^{+0.28}$ & $4.71_{-1.35}^{+1.78}$ & $1.70 \pm 0.06$ & $1.10_{-0.13}^{+0.14}$ & $\ldots$ & $0.660_{-0.016}^{+0.017}$ & $\ldots$ & $20.5 \pm 1.0$ & $4.90_{-0.36}^{+0.39}$ & $3.16_{-0.38}^{+0.41}$ & $6.19_{-0.95}^{+1.12}$ & $0.079_{-0.007}^{+0.008}$ & $0.051_{-0.008}^{+0.009}$ \\
\hline 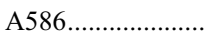 & $1.60_{-0.15}^{+0.19}$ & $6.99_{-2.04}^{+2.40}$ & $1.71 \pm 0.05$ & $1.50_{-0.21}^{+0.23}$ & & $0.627_{-0.014}^{+0.015}$ & & $31.9 \pm 1.4$ & $2.46_{-0.17}^{+0.18}$ & $2.15_{-0.31}^{+0.35}$ & $2.52_{-0.38}^{+0.46}$ & $0.098_{-0.009}^{+0.010}$ & $0.085_{-0.013}^{+0.016}$ \\
\hline 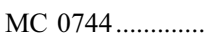 & $1.19_{-0.13}^{+0.24}$ & $5.40_{-2.47}^{+3.84}$ & $11.9_{-1.7}^{+2.1}$ & $12.0_{-2.2}^{+2.7}$ & $3.02_{-0.67}^{+0.83}$ & $0.584_{-0.049}^{+0.072}$ & $0.930_{-0.012}^{+0.009}$ & $22.2_{-5.6}^{+7.1}$ & $2.88_{-0.21}^{+0.24}$ & $2.87_{-0.31}^{+0.31}$ & $1.82_{-0.26}^{+0.31}$ & $0.158_{-0.012}^{+0.013}$ & $0.157_{-0.022}^{+0.029}$ \\
\hline 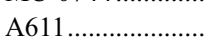 & $1.49_{-0.10}^{+0.12}$ & $5.08_{-1.01}^{+1.18}$ & $3.28_{-0.61}^{+1.00}$ & $3.12_{-0.66}^{+0.94}$ & $4.59_{-1.52}^{+2.89}$ & $0.602_{-0.011}^{+0.049}$ & $0.570_{-0.097}^{+0.083}$ & $23.3_{-1.6}^{+2.5}$ & $2.55_{-0.11}^{+0.12}$ & $2.40_{-0.28}^{+0.29}$ & $2.10_{-0.19}^{+0.26}$ & $0.122 \pm 0.007$ & $0.114 \pm 0.014$ \\
\hline A665... & $2.11_{-0.20}^{+0.24}$ & $35_{-0.64}^{+0.71}$ & $0.82_{-0.05}^{+0.06}$ & $0.91 \pm 0.11$ & $4.16_{-1.23}^{+1.81}$ & $0.661 \pm 0.014$ & $0.077_{-0.056}^{+0.073}$ & $65.2 \pm 2.0$ & $2.79 \pm 0.12$ & $3.09_{-0.31}^{+0.30}$ & $2.35_{-0.19}^{+0.20}$ & $0.119 \pm 0.005$ & $0.131 \pm 0.015$ \\
\hline A697... & $12_{-0.24}^{+0.28}$ & $\begin{array}{l}-1.34 \\
-0.95 \\
0.95\end{array}$ & $0.98 \pm 0.02$ & $0.92_{-0.15}^{+0.18}$ & & $0.593_{-0.011}^{+0.012}$ & $\ldots$ & $42.2 \pm 1.5$ & $4.68_{-0.38}^{+0.35}$ & $4.38_{-0.65}^{+0.63}$ & $3.57_{-0.51}^{+0.52}$ & $0.131_{-0.008}^{+0.010}$ & $0.122_{-0.021}^{+0.028}$ \\
\hline A773... & $1.56_{-0.13}^{+0.19}$ & $\begin{array}{r}-0.49 \\
+1.18 \\
-1.18\end{array}$ & $0.93 \pm 0.02$ & $1.31_{-0.15}^{+0.18}$ & & $0.586 \pm 0.016$ & & $41.7 \pm 1.9$ & $3.06 \pm 0.18$ & $4.34_{-0.46}^{+0.50}$ & $2.13_{-0.22}^{+0.24}$ & $0.144_{-0.007}^{+0.008}$ & $0.204_{-0.025}^{+0.029}$ \\
\hline Zw $3146 \ldots \ldots \ldots \ldots . . . .$. & $2.03_{-0.06}^{+0.07}$ & $\begin{array}{l}1.10 \\
+0.19 \\
-0.19\end{array}$ & $15.7 \pm 0.5$ & $14.2_{-1.6}^{+1.7}$ & $4.62_{-0.19}^{+0.18}$ & $0.668 \pm 0.006$ & $0.882 \pm 0.004$ & $26.0 \pm 0.9$ & $4.71 \pm 0.10$ & $4.25_{-0.43}^{+0.42}$ & $3.85_{-0.18}^{+0.19}$ & $0.122 \pm 0.004$ & $0.110_{-0.011}^{+0.012}$ \\
\hline MS $1054 \ldots \ldots \ldots . . .$. & $1.94_{-0.33}^{+0.37}$ & $90_{-0.30}^{+0.63}$ & $0.55 \pm 0.01$ & $0.53_{-0.07}^{+0.08}$ & $\ldots$ & $1.743_{-0.236}^{+0.188}$ & $\ldots$ & $86.1_{-8.3}^{+6.1}$ & $1.06 \pm 0.25$ & $1.03 \pm 0.16$ & $1.00_{-0.29}^{+0.34}$ & $0.106_{-0.010}^{+0.009}$ & $0.102_{-0.018}^{+0.020}$ \\
\hline MS $1137 \ldots \ldots \ldots \ldots \ldots . . .$. & $0.96_{-0.10}^{+0.15}$ & $12_{-3.34}^{+4.95}$ & $1.70 \pm 0.09$ & $2.25 \pm 0.32$ & $\ldots$ & $0.680_{-0.039}^{+0.041}$ & $\ldots$ & $14.6_{-12}^{+1.3}$ & $1.27_{-0.10}^{+0.11}$ & $1.69 \pm 0.17$ & $1.17_{-0.22}^{+0.27}$ & $0.109_{-0.012}^{+0.014}$ & $0.144_{-0.027}^{+0.032}$ \\
\hline MC $1149 \ldots \ldots \ldots \ldots$ & $1.96_{-0.12}^{+0.14}$ & $\begin{array}{l}5.37 \\
-0.67\end{array}$ & $0.68 \pm 0.02$ & $0.53 \pm 0.06$ & & $0.625_{-0.017}^{+0.019}$ & & $39.3_{-2.0}^{+2.1}$ & $3.03_{-0.28}^{+0.24}$ & $2.36_{-0.22}^{+0.21}$ & $2.82_{-0.40}^{+0.36}$ & $0.107_{-0.005}^{+0.007}$ & $0.084_{-0.011}^{+0.011}$ \\
\hline$\ldots \ldots . .$. & $4_{-0.13}^{+0.12}$ & $\begin{array}{l}-1.87 \\
-1.80\end{array}$ & $4.78_{-0.47}^{+0.61}$ & $5.40_{-1.03}^{+1.09}$ & $5.86_{-1.20}^{+1.14}$ & $0.519_{-0.009}^{+0.010}$ & $0.752 \pm 0.021$ & $36.6_{-2.8}^{+2.9}$ & $2.85_{-0.18}^{+0.10}$ & $3.18_{-0.47}^{+0.51}$ & $2.21_{-0.27}^{+0.15}$ & $0.129_{-0.004}^{+0.009}$ & $0.146_{-0.018}^{+0.020}$ \\
\hline$\ldots \ldots .$. & & $76_{-3.51}^{+4.80}$ & $2.49_{-0.14}^{-0.416}$ & $1.88_{-0.33}^{+0.43}$ & & $0.704_{-0.042}^{+0.049}$ & & $15.5_{-1.5}^{+1.6}$ & $4.17_{-0.52}^{+0.44}$ & $3.15_{-0.32}^{+0.31}$ & $4.69 \pm 1.28$ & $0.089_{-0.012}^{+0.0018}$ & $0.067_{-0.017}^{+0.028}$ \\
\hline MC $1311 \ldots$ & $1.25_{-0.13}^{+0.52}$ & $.44_{-7.35}^{+9.71}$ & $4.23_{-0.29}^{+0.39}$ & $4.70_{-0.95}^{+1.20}$ & & $0.615_{-0.022}^{+0.025}$ & & $9.2_{-7.1}^{+7.2}$ & $2.22_{-0.19}^{+0.17}$ & $2.46_{-0.37}^{+0.39}$ & $2.00_{-0.42}^{+0.41}$ & $0.111_{-0.012}^{+0.018}$ & $0.123_{-0.028}^{+0.038}$ \\
\hline A1689........ & $1.88 \pm 0.07$ & $56_{-0.73}^{+1.07}$ & $4.13_{-0.10}^{+0.11}$ & $4.35_{-0.37}^{+0.36}$ & $21.8_{-1.1}^{+1.5}$ & $0.889_{-0.041}^{+0.052}$ & $0.867 \pm 0.004$ & $104_{-6}^{+8.1}$ & $5.56 \pm 0.12$ & $5.85_{-0.49}^{+0.46}$ & $4.48_{-0.22}^{+0.26}$ & $0.124 \pm 0.004$ & $0.130_{-0.009}^{+0.010}$ \\
\hline R1347 ..................... & $2.10_{-0.12}^{+0.07}$ & & $25.3_{-0.6}^{+0.5}$ & $16.9_{-1.8}^{+1.9}$ & $3.88_{-0.13}^{+0.12}$ & $0.629 \pm 0.008$ & $0.942_{-0.004}^{+0.003}$ & $22.9_{-1.3}^{+1.4}$ & $9.55_{-0.47}^{+0.26}$ & $6.40_{-0.58}^{+0.60}$ & $7.96_{-0.87}^{+0.50}$ & $0.120_{-0.004}^{+0.008}$ & $0.081_{-0.009}^{+0.012}$ \\
\hline 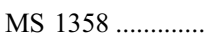 & $1.83 \pm 0.15$ & $\begin{array}{l}-0.42 \\
+0.53\end{array}$ & $10.2_{-0.5}^{+0.0}$ & $8.60_{-1.18}^{+1.26}$ & $3.30_{-0.24}^{+0.23}$ & $0.676_{-0.017}^{+0.016}$ & $0.934 \pm 0.003$ & $37.4_{-2.0}^{+1.8}$ & $2.82 \pm 0.18$ & $2.39_{-0.28}^{+0.30}$ & $3.14_{-0.41}^{+0.44}$ & $0.090_{-0.006}^{+0.007}$ & $0.078_{-0.012}^{+0.013}$ \\
\hline A1835 .................... & $1.95_{-0.40}^{+0.46}$ & $\begin{array}{l}+0.13 \\
-0.09\end{array}$ & $12.6 \pm 0.2$ & $12.5_{-0.8}^{+0.6}$ & $9.34_{-0.22}^{+0.21}$ & $0.797_{-0.013}^{+0.009}$ & $0.940 \pm 0.001$ & $63.6_{-1.8}^{+1.30}$ & $6.49_{-0.33}^{+0.30}$ & $6.41_{-0.41}^{+0.43}$ & $5.81_{-0.89}^{+0.88}$ & $0.112_{-0.019}^{+0.018}$ & $0.108_{-0.016}^{+0.015}$ \\
\hline MC $1423 \ldots \ldots \ldots \ldots$ & $1.30_{-0.04}^{+0.06}$ & $43_{-0.47}^{+0.45}$ & $16.7_{-0.3}^{+0.4}$ & $16.7_{-2.5}^{+2.7}$ & $4.08_{-0.14}^{+0.15}$ & $0.701_{-0.019}^{+0.024}$ & $0.976 \pm 0.002$ & $36.0_{-1.5}^{+1.4}$ & $2.56_{-0.08}^{+0.10}$ & $2.56_{-0.37}^{+0.36}$ & $2.08_{-0.15}^{+0.18}$ & $0.123_{-0.006}^{+0.005}$ & $0.122_{-0.019}^{+0.022}$ \\
\hline A1914...................... & $1.89_{-0.09}^{+0.12}$ & $85_{-1.57}^{+1.73}$ & $1.49_{-0.02}^{+0.03}$ & $1.27 \pm 0.11$ & $5.57_{-2.90}^{+6.60}$ & $0.890_{-0.011}^{+0.012}$ & $0.009_{-0.008}^{+0.021}$ & $67.7 \pm 1.0$ & $5.02_{-0.13}^{+0.16}$ & $4.29_{-0.38}^{+0.42}$ & $4.82_{-0.34}^{+0.46}$ & $0.104_{-0.006}^{+0.005}$ & $0.089 \pm 0.008$ \\
\hline A1995 ..................... & $2.62_{-0.39}^{+0.75}$ & $70_{-0.71}^{+0.75}$ & $0.90 \pm 0.01$ & $1.09 \pm 0.08$ & $\ldots$ & $0.916_{-0.023}^{+0.024}$ & $\ldots$ & $50.3_{-1.4}^{+1.5}$ & $3.79 \pm 0.20$ & $4.56_{-0.37}^{+0.35}$ & $4.42_{-0.54}^{+0.62}$ & $0.086 \pm 0.007$ & $0.103_{-0.012}^{+0.013}$ \\
\hline A2111 ................... & $2.04_{-0.44}^{+0.59}$ & $21_{-1.22}^{+2.36}$ & $0.55 \pm 0.03$ & $0.57_{-0.12}^{+0.14}$ & & $0.596_{-0.029}^{+0.032}$ & & $49.9_{-4.3}^{+1.5}$ & $2.31_{-0.37}^{+0.35}$ & $2.39 \pm 0.45$ & $2.34_{-0.59}^{+0.64}$ & $0.099_{-0.010}^{+0.013}$ & $0.102_{-0.026}^{+0.032}$ \\
\hline me & $2.89_{-0.23}^{+0.34}$ & & $0.94 \pm 0.01$ & 0.90 & $4.61_{-1.15}^{+1.81}$ & $0.559_{-0.005}^{+0.006}$ & $0.013_{-0.006}^{+0.014}$ & $67.0_{-0.9}^{+1.2 .}$ & $8.62_{-0.54}^{+0.49}$ & $8.21_{-0.72}^{+0.66}$ & $5.40_{-0.55}^{+0.53}$ & $0.160_{-0.006}^{+0.000}$ & $0.152_{-0.021}^{+0.019}$ \\
\hline A2204.......... & $2.43_{-0.12}^{+0.21}$ & $25_{-0.44}^{-0.33}$ & $21.1 \pm 0.4$ & $21.2_{-1.7}^{+1.9}$ & $7.93_{-0.30}^{+0.34}$ & $0.741_{-0.027}^{+0.028}$ & $0.957 \pm 0.003$ & $68.8_{-1.9}^{+2.9}$ & $5.00_{-0.22}^{+0.29}$ & $5.03_{-0.43}^{+0.47}$ & $5.79_{-0.55}^{+1.00}$ & $0.086_{-0.008}^{+0.006}$ & $0.086_{-0.011}^{+0.010}$ \\
\hline A2218 & $1.83_{-0.15}^{+0.20}$ & $4.27_{-1.14}^{+1.34}$ & $0.72 \pm 0.01$ & $0.81_{-0.09}^{+0.10}$ & $\ldots$ & $0.746 \pm 0.015$ & $\ldots$ & $69.1 \pm 1.8$ & $3.17 \pm 0.14$ & $3.56_{-0.42}^{+0.48}$ & $3.07_{-0.28}^{+0.35}$ & $0.103 \pm 0.005$ & $0.116_{-0.012}^{+0.011}$ \\
\hline R1716 ...................... & $1.11_{-0.16}^{+0.38}$ & & $1.53_{-0.16}^{+0.20}$ & & $\ldots$ & $0.540_{-0.043}^{+0.052}$ & & $11.2_{-1.7}^{+2.1}$ & $1.33_{-0.29}^{+0.22}$ & $1.16_{-0.27}^{+0.26}$ & $1.39_{-0.54}^{+0.49}$ & $0.095_{-0.014}^{+0.026}$ & $0.083_{-0.027}^{+0.040}$ \\
\hline A2259.................. & $1.47_{-0.17}^{+0.23}$ & $.74_{-1.37}^{+1.76}$ & $0.94 \pm 0.04$ & $1.39_{-0.34}^{+0.40}$ & & $0.557_{-0.019}^{+0.021}$ & & $40.5_{-2.7}^{+2.8}$ & $1.95 \pm 0.13$ & $1.99_{-0.55}^{+0.56}$ & $1.54_{-0.19}^{+0.20}$ & $0.126_{-0.008}^{+0.009}$ & $0.131_{-0.038}^{+0.037}$ \\
\hline A2261..................... & $1.54_{-0.14}^{+0.15}$ & $86_{-1.53}^{+2.16}$ & $3.93_{-0.29}^{+0.39}$ & $4.14_{-0.59}^{+0.64}$ & $11.7_{-2.1}^{+1.9}$ & $0.659_{-0.032}^{+0.043}$ & $0.803_{-0.039}^{+0.034}$ & $44.6_{-6.5}^{+10.2}$ & $3.34_{-0.23}^{+0.22}$ & $3.50_{-0.38}^{+0.44}$ & $2.55_{-0.38}^{+0.40}$ & $0.131_{-0.011}^{+0.013}$ & $0.138_{-0.019}^{+0.022}$ \\
\hline MS $2053 \ldots \ldots \ldots \ldots$ & $0.92 \pm 0.16$ & $8_{-4.06}^{+6.41}$ & $1.15_{-0.11}^{+0.14}$ & $1.77_{-0.45}^{+0.60}$ & $\cdots$ & $0.552_{-0.055}^{+0.051}$ & $\ldots$ & $12.5_{-2.1}^{+1.8}$ & $0.99_{-0.21}^{+0.18}$ & $1.53_{-0.23}^{+0.25}$ & $0.84_{-0.33}^{+0.36}$ & $0.117_{-0.021}^{+0.035}$ & $0.183_{-0.056}^{+0.082}$ \\
\hline MC $2129 \ldots \ldots \ldots \ldots . . . .$. & & & $1.75 \pm 0.07$ & $1.69_{-0.23}^{+0.27}$ & & $0.602_{-0.020}^{+0.022}$ & & $18.6_{-1.1}^{+1.2}$ & $3.53 \pm 0.23$ & $3.41 \pm 0.40$ & $2.35_{-0.30}^{+0.32}$ & $0.150_{-0.010}^{+0.012}$ & $0.145_{-0.022}^{+0.027}$ \\
\hline 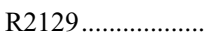 & & & $14.1_{-1.2}^{+1.6}$ & $11.3_{-2.0}^{+1.9}$ & $3.44 \pm 0.44$ & $0.584_{-0.015}^{+0.016}$ & $0.910_{-0.010}^{+0.009}$ & $25.7_{-2.6}^{+2.1}$ & $2.80_{-0.19}^{+0.16}$ & $2.23_{-0.34}^{+0.31}$ & $2.29_{-0.32}^{+0.30}$ & $0.122_{-0.008}^{+0.010}$ & $0.098_{-0.018}^{+0.019}$ \\
\hline MC $2214 \ldots \ldots \ldots \ldots$ & $1.64_{-0.20}^{-0.15}$ & $4.95_{-1.69}^{+2.75}$ & $1.46 \pm 0.05$ & $1.58_{-0.17}^{+0.20}$ & $\ldots$ & $0.606 \pm 0.019$ & $\ldots$ & $22.3 \pm 1.3$ & $4.15_{-}^{+}$ & $4.49_{-0.38}^{+0.34}$ & $3.48_{-0.70}^{+0.32}$ & $0.119_{-0.011}^{+0.0015}$ & $0.129_{-0.019}^{+0.018}$ \\
\hline MC $2228 \ldots \ldots \ldots \ldots$ & $1.94_{-0.22}^{+0.20}$ & $1.92_{-0.83}^{+1.05}$ & $1.31_{-0.06}^{+0.07}$ & $1.58_{-0.22}^{+0.28}$ & $\cdots$ & $0.518 \pm 0.013$ & $\cdots$ & $21.4_{-1.4}^{+1.5}$ & $2.99_{-0.45}^{+0.34}$ & $3.63_{-0.34}^{+0.30}$ & $2.19_{-0.54}^{+0.49}$ & $0.136_{-0.012}^{+0.018}$ & $0.165_{-0.027}^{+0.040}$ \\
\hline
\end{tabular}


TABLE 6

Model Parameters, Cluster Masses, and Gas Mass Fractions: Isothermal $\beta$-Model Constraints Derived from SZE IMAging Data

\begin{tabular}{|c|c|c|c|c|c|}
\hline Cluster & $\begin{array}{c}\theta_{c} \\
(\operatorname{arcsec})\end{array}$ & $\begin{array}{c}\Delta T_{0} \\
(\mathrm{mK})\end{array}$ & $\begin{array}{c}M_{\text {gas }}\left(r_{2500}\right) \\
\left(10^{13} M_{\odot}\right)\end{array}$ & $\begin{array}{c}M_{\text {total }}\left(r_{2500}\right) \\
\left(10^{14} M_{\odot}\right)\end{array}$ & $f_{\text {gas }}\left(r_{2500}\right)$ \\
\hline CL $0016 \ldots \ldots \ldots \ldots \ldots \ldots \ldots$ & $52.0_{-8.1}^{+9.0}$ & $-1.449_{-0.094}^{+0.088}$ & $4.65_{-0.39}^{+0.42}$ & $2.83 \pm 0.32$ & $0.165_{-0.027}^{+0.033}$ \\
\hline A267............................ & $38.6_{-12.8}^{+15.8}$ & $-0.735_{-0.099}^{+0.084}$ & $2.74_{-0.57}^{+0.68}$ & $1.99_{-0.23}^{+0.25}$ & $0.137_{-0.038}^{+0.054}$ \\
\hline A $370 \ldots \ldots \ldots \ldots \ldots \ldots \ldots \ldots \ldots \ldots \ldots \ldots$ & $21.5_{-7.4}^{+9.3}$ & $-1.072_{-0.244}^{+0.149}$ & $2.58_{-0.47}^{+0.47}$ & $3.18 \pm 0.22$ & $0.081_{-0.016}^{+0.028}$ \\
\hline MS $0451 \ldots \ldots \ldots$ & $31.4_{-6.4}^{+7.4}$ & $-1.610_{-0.112}^{+0.100}$ & $5.03_{-0.71}^{+0.83}$ & $3.46_{-0.31}^{+0.32}$ & $0.145_{-0.030}^{+0.039}$ \\
\hline MC $0647 \ldots \ldots \ldots \ldots \ldots \ldots \ldots$ & $19.1_{-6.6}^{+0.4}$ & $-1.390_{-0.242}^{+0.151}$ & $2.81_{-0.57}^{+0.71}$ & $6.21_{-0.74}^{+0.81}$ & $0.045_{-0.012}^{+0.030}$ \\
\hline $\mathrm{A} 665 \ldots \ldots \ldots \ldots$ & $56.8_{-175}^{+63.6}$ & $-0.801_{-0.105}^{+0.083}$ & $2.43_{-0.53}^{+0.71}$ & $2.77_{-0.29}^{+0.21}$ & $0.087_{-0.023}^{+0.038}$ \\
\hline A773 & $30.9_{-9.8}^{+11.5}$ & $-0.985_{-0.128}^{+0.109}$ & $2.56_{-0.54}^{+0.67}$ & $3.12_{-0.22}^{+0.23}$ & $0.082_{-0.020}^{+0.027}$ \\
\hline 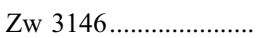 & $29.4_{-9.9}^{+11.9}$ & $-1.223_{-0.242}^{+0.1179}$ & $3.95_{-0.73}^{+0.08}$ & $3.43_{-019}^{+0.17}$ & $0.115_{-0.024}^{+0.030}$ \\
\hline 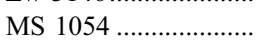 & $54.5_{-10.4}^{+11.4}$ & $-1.483_{-0.253}^{+0.191}$ & $1.17_{-0.15}^{+0.16}$ & $0.62_{-0.16}^{+0.19}$ & $0.187_{-0.054}^{+0.024}$ \\
\hline 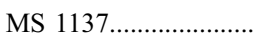 & $12.2_{-4.4}^{+8.4}$ & $-1.021_{-0.441}^{+0.303}$ & $2.09_{-0.36}^{+0.38}$ & $0.96_{-0.14}^{+0.15}$ & $0.221_{-0.051}^{+0.064}$ \\
\hline 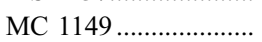 & $53.2_{-16.6}^{+19.1}$ & $-1.181_{-0.160}^{+0.127}$ & $3.00_{-0.37}^{+0.46}$ & $2.17_{-0.53}^{-0.14}$ & $0.139_{-0.038}^{+0.059}$ \\
\hline CL $1226 \ldots \ldots \ldots \ldots \ldots \ldots \ldots$ & $11.1_{-4.5}^{+8.2}$ & $-2.171_{-1164}^{+0.677}$ & $2.82_{-0.51}^{+0.63}$ & $5.08_{-0.88}^{+1.05}$ & $0.056_{-0.017}^{+0.028}$ \\
\hline A1689 & $25.2_{-5.9}^{+6.9}$ & $-1.749_{-0.13}^{+0.171}$ & $3.57_{-0.57}^{+0.69}$ & $5.21 \pm 0.23$ & $0.069_{-0.012}^{+0.015}$ \\
\hline $\mathrm{R} 1347 \ldots .$. & $28.7_{-8.5}^{+10.5}$ & $-2.235_{-0.429}^{+0.316}$ & $5.88_{-0.85}^{+0.97}$ & $8.35 \pm 0.56$ & $0.070_{-0.013}^{+0.012}$ \\
\hline A1835...... & $50.1_{-8.2}^{+9.2}$ & $-1.590_{-0.113}^{+0.112}$ & $6.20_{-0.82}^{+0.95}$ & $5.38_{-0.31}^{+0.34}$ & $0.115_{-0.020}^{+0.024}$ \\
\hline A1914........ & $29.4_{-8.9}^{+11.2}$ & $-1.776_{-0.317}^{+0.200}$ & $4.33_{-0.75}^{+0.96}$ & $4.69_{-0.16}^{+0.18}$ & $0.092_{-0.017}^{+0.023}$ \\
\hline A1995........ & $34.6_{-54}^{+5.5}$ & $-1.051_{-0.063}^{+0.062}$ & $4.49_{-0.57}^{+0.63}$ & $3.63_{-0.21}^{+0.10}$ & $0.124_{-0.020}^{+0.024}$ \\
\hline 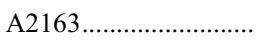 & $102.7_{-15.1}^{+17.0}$ & $-1.898_{-0.336}^{+0.276}$ & $7.57_{-1.38}^{+1.61}$ & $6.17_{-0.44}^{+0.38}$ & $0.123_{-0.028}^{+0.036}$ \\
\hline 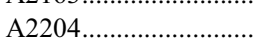 & $100.2_{-18.5}^{+20.1}$ & $-1.938_{-0.382}^{+0.336}$ & $8.96_{-2.13}^{+2.38}$ & $4.95_{-0.41}^{+0.44}$ & $0.180_{-0.051}^{+0.028}$ \\
\hline 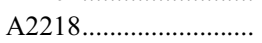 & $79.0_{-12.4}^{+13.6}$ & $-1.059_{-0.176}^{+0.143}$ & $5.34_{-1.03}^{+1.23}$ & $2.95_{-0.20}^{+0.41}$ & $0.181_{-0.042}^{+0.055}$ \\
\hline A2261......................... & $20.9_{-7.5}^{+9.8}$ & $-1.179_{-0.239}^{+0.140}$ & $2.46_{-0.54}^{+0.75}$ & $2.91_{-0.23}^{+0.20}$ & $0.084_{-0.021}^{+0.041}$ \\
\hline 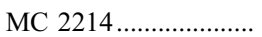 & $27.3_{-7.1}^{+8.3}$ & $-1.518_{-0.227}^{+0.160}$ & $4.37_{-0.55}^{+0.60}$ & $3.87_{-0.38}^{+0.41}$ & $0.113_{-0.022}^{+0.028}$ \\
\hline 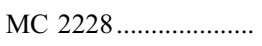 & $47.3_{-15.5}^{+18.7}$ & $-1.315_{-0.164}^{+0.137}$ & $3.91_{-0.56}^{+0.65}$ & $2.15_{-0.43}^{+0.40}$ & $0.182_{-0.049}^{+0.076}$ \\
\hline
\end{tabular}

model $(\S 4.1 .1)$. The X-ray and SZE data are fit jointly, with the cluster core radius and $\beta$-parameter linked between data sets, while the central surface brightness $S_{\mathrm{X} 0}$ and central SZE decrement $\Delta T_{0}$ are allowed to vary. The gas mass fractions are plotted as a function of redshift in Figure 3. X-ray surface brightness profiles and the best-fit $100 \mathrm{kpc}$ cut models are shown in the Appendix (Fig. 5) for the 38 clusters in our sample. The $\mathrm{X}$-ray surface brightness profiles are well described by the isothermal $\beta$-model beyond $100 \mathrm{kpc}$ from the cluster center.

In Table 5 we present X-ray and SZE gas mass fractions determined from the nonisothermal model ( $\S 4.1 .2)$. The X-ray and SZE data are again fit jointly with shape parameters linked, while the X-ray and SZE central density parameters are allowed to vary separately. The gas mass fractions are plotted as a function of redshift in Figure 3. Surface brightness profiles for the nonisothermal model are shown in Bonamente et al. (2006) and show consistently good fits to the cluster radial profiles out to $r_{2500}$.

In Table 6 we present SZE gas mass fractions determined from the SZE-only model $(\S 4.1 .3)$. We find that this model constrains $\Delta T_{0}$ and $\theta_{c}$ individually in 23 of our 38 clusters, and those 23 are shown in the table. Gas mass fractions derived with this method are plotted versus redshift in Figure 3.

The mean X-ray and SZE gas mass fractions for all three models are summarized in Table 7, where the uncertainties are statistical followed by systematic at $68 \%$ confidence. The statistical uncertainties include the effects of cluster asphericity, radio point sources, and the kinetic SZE, combined in quadrature; systematic uncertainties include errors associated with instrument calibration, X-ray background, and the assumptions of HSE and isothermality (see $\S 5$ and Table 3). The results show that the X-ray and SZE measurements of the mean gas mass fraction in clusters agree to better than $10 \%$ for the three ICM models considered.

\subsection{Effects of Cluster Cores on Derived Gas Mass Fractions}

The results summarized in Table 7 show that the X-ray- and SZE-derived gas mass fractions agree remarkably well as long as care is taken to account for the effect of the cluster core on the $\mathrm{X}$-ray emission. The results show that the core can be satisfactorily accounted for by either ignoring the core in fits to the X-ray data (the $100 \mathrm{kpc}$ cut model) or modeling the ICM with the nonisothermal double $\beta$-model.

It is also noteworthy that the gas mass fraction results derived from spatial fits to only the SZE data including the core also agree remarkably well with the above models. This indicates that the SZE is not very sensitive to the cluster core and is in line with expectations, since the SZE is a probe of the ICM pressure.

The effect of cluster cores on the gas mass fractions is further investigated by comparing results for cool-core and non-coolcore subsamples. There is a population of clusters in our sample with bright and sharply peaked cores (see the Appendix), including A586, MC 0744, Zw 3146, A1413, MC 1311, A1689, R1347, MS 1358, A1835, MC 1423, A2204, A2261, and R2129. These

TABLE 7

Mean Gas Mass Fractions

\begin{tabular}{|c|c|c|}
\hline Method Used in Spatial Analysis & $f_{\text {gas }}^{\mathrm{X}-\text { ray }}$ & $f_{\mathrm{gas}}^{\mathrm{SZE}}$ \\
\hline 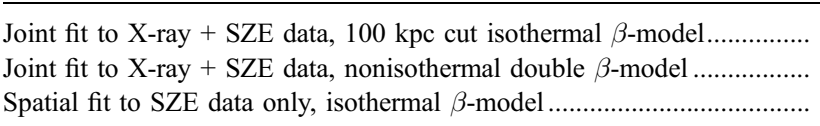 & $\begin{array}{c}0.110 \pm 0.003_{-0.018}^{+0.006} \\
0.119 \pm 0.003_{-0.014}^{+0.007} \\
\quad \cdots\end{array}$ & $\begin{array}{l}0.116 \pm 0.005_{-0.026}^{+0.009} \\
0.121 \pm 0.005_{-0.0096}^{+0.009} \\
0.120 \pm 0.009_{-0.027}^{+0.009}\end{array}$ \\
\hline
\end{tabular}


TABLE 8

Comparison of Cool-Core and Non-Cool-Core Results

\begin{tabular}{|c|c|c|c|}
\hline Analysis Method & Data Set & Cool-Core & Non-Cool-Core \\
\hline \multirow[t]{2}{*}{ Joint fit X-ray + SZE $100 \mathrm{kpc}$ cut isothermal $\beta$-model $\ldots \ldots \ldots \ldots \ldots \ldots . . . .}$. & $\mathrm{X}$-ray & $0.110 \pm 0.004$ & $0.109 \pm 0.004$ \\
\hline & SZE & $0.107 \pm 0.007$ & $0.120 \pm 0.006$ \\
\hline \multirow{2}{*}{ Joint fit X-ray + SZE nonisothermal double $\beta$-model................... } & $\mathrm{X}$-ray & $0.118 \pm 0.005$ & $0.120 \pm 0.005$ \\
\hline & SZE & $0.113 \pm 0.007$ & $0.125 \pm 0.007$ \\
\hline 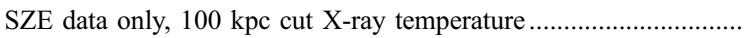 & SZE & $0.098 \pm 0.015$ & $0.129 \pm 0.011$ \\
\hline \multirow[t]{2}{*}{ Joint fit X-ray + SZE no-cut isothermal $\beta$-model $\ldots \ldots \ldots \ldots \ldots \ldots \ldots \ldots \ldots \ldots \ldots \ldots \ldots \ldots$} & X-ray & $0.139 \pm 0.009$ & $0.106 \pm 0.004$ \\
\hline & SZE & $0.158 \pm 0.014$ & $0.118 \pm 0.006$ \\
\hline SZE data only, no-cut X-ray temperature ${ }^{\mathrm{a}} .$. & SZE & $0.148 \pm 0.037$ & $0.123 \pm 0.009$ \\
\hline
\end{tabular}

${ }^{a}$ The large statistical uncertainties for this subsample reflect the small sample sizes: six clusters in the cool-core case and 17 in the non-cool-core case.

clusters have central cooling times (calculated with the cores included in the X-ray data) $t_{\text {cool }} \approx 3 k_{\mathrm{B}} T_{\mathrm{X}} \mu_{\mathrm{H}} / 2 \Lambda_{e \mathrm{H}} n_{e} \mu_{\text {total }}<0.5 t_{\text {Hubble }}$, and we refer to this subsample as the cool-core sample because the sharply peaked X-ray emission is indicative of strong radiative cooling in the cluster core. The results of a comparison of coolcore and non-cool-core weighted mean gas mass fractions are shown in Table 8, where in addition to the three main ICM models considered above, we include results from two additional naive models that make no effort to compensate for the cluster cores: (1) a no-cut isothermal $\beta$-model fit jointly to the full X-ray and SZE data sets and (2) a spatial model fit to only the SZE data as before but using the $\mathrm{X}$-ray temperature derived from all of the $\mathrm{X}$-ray data instead of the data with the central $100 \mathrm{kpc}$ excluded.

Apart from a small $<10 \%$ offset in the X-ray results, the derived gas mass fractions for the $100 \mathrm{kpc}$ cut and nonisothermal models for the cool-core and non-cool-core clusters are in excellent agreement. Figure 3 also demonstrates this agreement, as there is no systematic offset seen between the cool-core (triangles) and non-cool-core (squares) clusters. The results for the naive models clearly show the importance of accounting for the core in the cool-core subsample; large offsets are found for the derived gas mass fraction of the cool-core subsample, while the same naive models provide perfectly consistent results for the non-coolcore subsample. The primary cause of the biased results of the naive models for the cool-core clusters is the poor spatial fits and the low $T_{\mathrm{X}}$. A depressed $T_{\mathrm{X}}$ results in a lower derived total cluster mass as well as a higher SZE gas mass.

\subsection{Constraints on Clumping in the Intracluster Gas}

The comparison of the X-ray and SZE gas mass fraction measurements shows that the results are in good agreement. These results suggest a clumping factor of the ICM consistent with zero. The high-resolution Chandra data allow one to remove any visible clumps before the data are fit, and the agreement between $\mathrm{X}$-ray and SZE gas mass fraction measurements indicates that any clumps below the noise level of the data have a negligible effect on the mass measurements. Further indications in favor of a negligible clumping factor are discussed in $\S 5.9$.

\subsection{Comparison with Previous Studies}

Finally, we compare our X-ray and SZE gas mass measurements to the results from previous studies in the literature. We first compare our X-ray results with Chandra results obtained using a different analysis technique (Allen et al. 2002, 2004), ${ }^{10}$ in which

${ }^{10}$ Although mean gas mass fraction results for other cluster samples are available in the literature (e.g., Evrard 1997; Mohr et al. 1999; Ettori et al. 2003; Sanderson et al. 2003), the detailed comparisons with our results are nontrivial as they are often calculated at different radii and assume different cosmologies. the cluster mass is modeled assuming that the cluster gas is in hydrostatic equilibrium with a NFW potential and using a deprojected 3D gas temperature profile. These authors report mean gas mass fractions of $f_{\text {gas }}=0.113 \pm 0.005$ for a sample of six highly relaxed clusters (Allen et al. 2002) and $f_{\text {gas }}=0.117 \pm 0.002$ for a larger sample of 26 relaxed clusters (Allen et al. 2004); these results are in good agreement with our own. We also compare the results of eight clusters we have in common with the Allen et al. (2004) data set and find that the measurements of individual clusters are consistent with no systematic offset.

For the SZE gas mass fractions, we compare results of our SZEonly model fitting directly with the results of Grego et al. (2001). There are many similarities between our cluster sample and analysis method and those of Grego et al.; for instance, they use BIMA and OVRO data, and 13 of our 23 clusters have data in common with their sample. They also use an isothermal $\beta$-model to analyze the SZE data. But their approach differs somewhat from ours, in that they (1) use spectroscopic temperatures from the literature (generally from the Advanced Satellite for Cosmology and Astrophysics $[A S C A]$ ) and (2) measure the gas mass fraction at $65^{\prime \prime}$ from the cluster center, then scale the results to $r_{500}$ using relations calibrated from numerical simulations (Evrard et al. 1996; Evrard 1997). Despite these differences, Grego et al. measure a mean gas mass fraction of $f_{\text {gas }}=0.081_{-0.011}^{+0.009} h^{-1}$, which is equivalent to $0.116_{-0.016}^{+0.013}$ for $h=0.7$, in good agreement with our SZEderived gas mass fraction (see Table 7). Similarly, if we calculate gas mass fractions from our SZE-only model at $65^{\prime \prime}$ and scale to $r_{500}$ using the same relations, we obtain a weighted mean $f_{\text {gas }}$ of $0.086 \pm 0.007 h^{-1}$, in good agreement with the Grego et al. results. The cluster gas mass fraction results derived from the implied SZE for nearby clusters obtained from correlating known clusters with Wilkinson Microwave Anisotropy Probe (WMAP) data is also consistent with our results (Afshordi et al. 2005).

\section{CONSTRAINTS ON CLUSTER PHYSICS AND COSMOLOGY}

\subsection{The Cluster Baryon Budget}

Currently, the relation between $f_{\text {gas }}$ and the cosmological baryon fraction $\Omega_{B} / \Omega_{M}$ is not accurately known (Ettori et al. 2006). However, since recent CMB studies provide precise constraints on cosmological parameters (e.g., Spergel et al. 2006), we can use our $f_{\text {gas }}$ measurements to obtain constraints on the ratio

$$
\eta_{\text {gas }} \equiv \frac{f_{\text {gas }}}{\Omega_{B} / \Omega_{M}} .
$$

Combining our $f_{\text {gas }}$ results with the WMAP values of $\Omega_{B}$ and $\Omega_{M}$ $\left(\Omega_{B} h^{2}=0.0223_{-0.0009}^{+0.0007}, \Omega_{M} h^{2}=0.127_{-0.013}^{+0.007}\right)$ and the Hubble 
TABLE 9

Constraints on $\eta_{\text {gas }}$

\begin{tabular}{|c|c|c|}
\hline Method Used in Spatial Analysis & $\eta_{\mathrm{gas}}^{\mathrm{X}-\mathrm{ray}}$ & $\eta_{\mathrm{gas}}^{\mathrm{SZE}}$ \\
\hline Joint fit to X-ray + SZE data, $100 \mathrm{kpc}$ cut isothermal $\beta$-model.. & $0.65 \pm 0.03_{-0.10}^{+0.04}$ & $0.65_{-0.03}^{+0.02+0.05}$ \\
\hline Joint fit to X-ray + SZE data, nonisothermal double $\beta$-model .... & $0.71 \pm 0.03_{-0.09}^{+0.04}$ & $0.67 \pm 0.03_{-0.09}^{+0.05}$ \\
\hline Spatial fit to SZE data only, isothermal $\beta$-model ....................... & $\ldots$ & $0.64_{-0.03-0.14}^{+0.05+0.06}$ \\
\hline
\end{tabular}

Space Telescope measurement of $h=0.72 \pm 0.08$ (Freedman et al. 2001), we obtain the constraints on $\eta_{\text {gas }}$ given in Table 9, where uncertainties are again statistical followed by systematic at $68 \%$ confidence. Since the $f_{\text {gas }}$ data are calculated assuming $\Omega_{M}=$ $0.3, \Omega_{\Lambda}=0.7$, and $h=0.7$, very similar to the $W M A P$ constraints, no significant bias is expected in the results of Table 9. These results indicate that the ratio of the gas mass fraction in clusters within $r_{2500}$ to the cosmic baryon fraction, $f_{\text {gas }} /\left(\Omega_{B} / \Omega_{M}\right)$, is $0.68_{-0.16}^{+0.10}$, where the range includes statistical and systematic uncertainties, in accord with the conclusions reached by Afshordi et al. (2005). This ratio provides a benchmark for simulations of galaxy clusters. We note that recent numerical simulations that include radiative cooling and star formation have baryon budgets in line with our results, although they find that the gas tends to overcool in the cluster cores (Kravtsov et al. 2005).

\subsection{Cosmological Parameters}

Cluster gas mass fractions can provide strong constraints on cosmological parameters. The normalization of the cluster gas mass fraction depends sensitively on the ratio $\Omega_{B} / \Omega_{M}$ (e.g., White et al. 1993; David et al. 1995; Myers et al. 1997; Mohr et al. 1999; Ettori \& Fabian 1999; Grego et al. 2001; Allen et al. 2002), while the redshift evolution of the gas mass fraction depends sensitively on $\Omega_{M}, \Omega_{\Lambda}$, and even the equation of dark energy, $w$ (Sasaki 1996; Pen 1997; Allen et al. 2004). Methods involving the normalization, however, depend sensitively on a priori knowledge of the cluster baryon budget, which, as discussed in $\S 7.1$, is poorly known. By contrast, methods using the redshift evolution are powerful in that they are independent of the cluster baryon budget and even prior knowledge on other cosmological parameters such as $\Omega_{B}$ and $h$.

We therefore consider constraints on cosmological parameters from the redshift evolution of the cluster gas mass fraction. For parameter estimation, we follow the procedure described in Allen et al. (2004) and fit the data to the model,

$$
f_{\mathrm{gas}}^{\bmod }(z)=N\left[\frac{D_{A}\left(z, \Omega_{M}^{\mathrm{fid}}, \Omega_{\Lambda}^{\mathrm{fid}}\right)}{D_{A}\left(z, \Omega_{M}^{\bmod }, \Omega_{\Lambda}^{\mathrm{mod}}\right)}\right]^{[3 / 2(\mathrm{X}-\text { ray }), 1(\mathrm{SZE})]},
$$

where we assume a $\Lambda$ CDM cosmology with $\Omega_{M}=0.3$ and $\Omega_{\Lambda}=0.7$ as a fiducial cosmology, indicated by "fid." Using this model, we fit an arbitrary normalization $N$ and grid the variables $\Omega_{M}$ and $\Omega_{\Lambda}$ between 0 and 1.3 with the WMAP constraint $\Omega_{M}+$ $\Omega_{\Lambda}=1.003_{-0.017}^{+0.013}$ (Spergel et al. 2006). We cannot place useful constraints on $\Omega_{M}$ and $\Omega_{\Lambda}$ from the X-ray or SZE data without a prior on the total density parameter.

Marginalizing over the $W M A P$ total density prior, we find bestfit parameters of $\Omega_{M}=0.26_{-0.15}^{+0.24}$ and $\Omega_{\Lambda}=0.74_{-0.24}^{+0.15}$ from the $100 \mathrm{kpc}$ cut X-ray data and $\Omega_{M}=0.40_{-0.26}^{+0.51}$ and $\Omega_{\Lambda}=0.60_{-0.51}^{+0.26}$ from the isothermal $\beta$-model analysis. Similarly, the nonisothermal model returns $\Omega_{M}=0.40_{-0.20}^{+0.28}$ and $\Omega_{\Lambda}=0.60_{-0.28}^{+0.20}$ from X-rays and $\Omega_{M}=0.18_{-0.17}^{+0.27}$ and $\Omega_{\Lambda}^{-0.20}=0.82_{-0.27}^{+0.17}$ from SZE. Our results are consistent with independent tests, such as those of $W M A P$, and inconsistent with a matter-dominated universe $\left(\Omega_{M}=1, \Omega_{\Lambda}=0\right)$ model. In Figure 4 we show the $f_{\text {gas }}$ data, the best-fit model using the WMAP prior (solid line), the best-fit model without the WMAP prior (unconstrained; dashed line), and the model corresponding to two other fiducial cosmologies
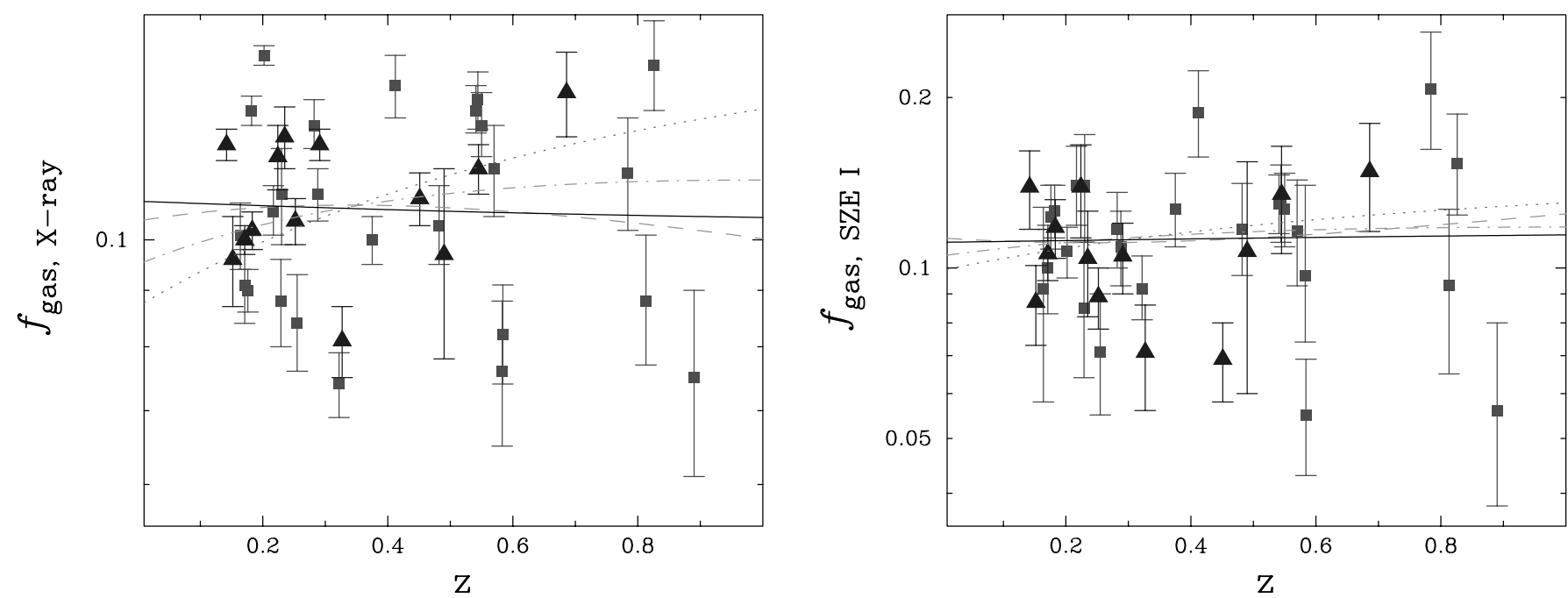

FIG. 4.-Same as Fig. 3, but for the $100 \mathrm{kpc}$ cut derived gas mass fractions and showing the best-fit models for different cosmologies. The solid lines show the best-fit

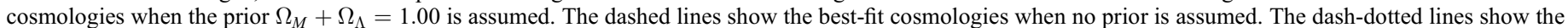

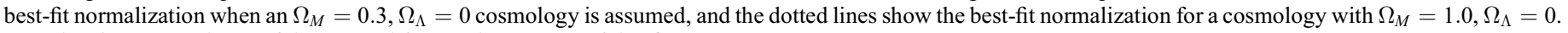
[See the electronic edition of the Journal for a color version of this figure.] 
(dotted line, $\Omega_{M}=1$ and $\Omega_{\Lambda}=0$; dash-dotted line, $\Omega_{M}=0.3$ and $\Omega_{\Lambda}=0$ ). Only the $100 \mathrm{kpc}$ cut results are plotted for simplicity. It is evident that the X-ray data disfavor the flat, matterdominated model. Interestingly, systematic uncertainty should be negligible compared to statistical uncertainty with this method, since most of the systematic uncertainties affect the normalization and do not introduce trends with redshift. Cosmological constraints from this method are therefore expected to improve as the cluster sample size is increased.

\section{CONCLUSIONS}

We have presented X-ray and SZE measurements of gas mass fractions for a sample of 38 massive galaxy clusters using Chandra X-ray observations and BIMA/OVRO SZE radio interferometric observations. Three models for the cluster gas distributions are used in the gas mass fraction derivations: (1) an isothermal $\beta$-model fit to the X-ray data at radii beyond $100 \mathrm{kpc}$ and to all of the SZE data, (2) a nonisothermal double $\beta$-model fit to all of the X-ray and SZE data, and (3) an isothermal $\beta$-model fit to the SZE spatial data with $\beta$ fixed at 0.7 and a spectral temperature from the $100 \mathrm{kpc}$ cut X-ray data.

The cluster gas distributions are well fit by the nonisothermal model out to $r_{2500}$. We have also demonstrated that the simple $100 \mathrm{kpc}$ cut isothermal model provides a good description of the intracluster medium (ICM) outside the cluster core for objects with a wide range of morphological properties.

For all three models, the mean gas mass fractions determined from the X-ray and SZE data are in excellent agreement. Specifically, with the isothermal $\beta$-model we find a mean X-ray gas mass fraction $f_{\text {gas }}(\mathrm{X}$-ray $)=0.110 \pm 0.003_{-0.018}^{+0.006}$ and a mean SZE gas mass fraction $f_{\text {gas }}(\mathrm{SZE})=0.116 \pm 0.005_{-0.026}^{+0.009}$, where uncertainties are statistical followed by systematic at $68 \%$ confidence. For the nonisothermal double $\beta$-model, the mean gas mass fractions are $f_{\text {gas }}$ (X-ray) $=0.119 \pm 0.003_{-0.014}^{+0.007}$ and $f_{\text {gas }}(\mathrm{SZE})=0.121 \pm 0.005_{-0.016}^{+0.009}$. For the SZE-only model, $f_{\text {gas }}(\mathrm{SZE})=0.120 \pm 0.009_{-0.027}^{+0.009}$.

It is noteworthy that the gas mass fractions derived from spatial fits to only the SZE data including the core also agree remarkably well with the other models. This indicates that the SZE is largely insensitive to structure in the cluster core. The lack of sensitivity to the core structure is expected, since the SZE is a probe of the gas pressure; this is promising for extracting cosmology from upcoming large-scale SZE cluster surveys (e.g., see Carlstrom et al. 2002).

To investigate the effect of the cluster cores on the results, we divided the cluster sample into two subsamples depending on whether or not the clusters exhibit cool cores. The three ICM models above lead to consistent gas mass fraction results for each subsample. However, when the subsamples are fit with naive ICM models that make no effort to account for the cluster cores, the resulting gas mass fractions for the cool-core subsample show large offsets from the above results, while the results for the non-coolcore clusters are consistent with the above results. This clearly indicates the importance of accounting for the cores in clusters that exhibit cool cores.

Understanding the baryon budget in clusters is one of the most important aspects of cluster physics. Our X-ray and SZE mea- surements indicate that the ratio of the gas mass fraction within $r_{2500}$ to the cosmic baryon fraction, $f_{\text {gas }} /\left(\Omega_{B} / \Omega_{M}\right)$, is $0.68_{-0.16}^{+0.10}$, where the range includes statistical and systematic uncertainties at $68 \%$ confidence. This result is in accord with the conclusions reached by Afshordi et al. (2005). The measurement provides a benchmark for simulations of galaxy clusters. We note that recent numerical simulations that include radiative cooling and star formation have baryon budgets in line with our measurements, although they find that the gas tends to overcool in the cluster cores (Kravtsov et al. 2005).

Our results also have implications for cosmological constraints based on measurements of cluster gas mass fractions. Constraints from the normalization of the gas mass fraction depend sensitively on a priori knowledge of the cluster baryon budget $\eta_{\text {gas }}$, which, as discussed above, is still somewhat uncertain. However, a cosmological test based on the redshift evolution of the gas mass fraction is independent of the cluster baryon budget and even prior knowledge of cosmological parameters such as $\Omega_{B}$ and $h$. Marginalizing over the $W M A P$ total density prior, we use this method and find best-fit parameters of $\Omega_{M}=0.26_{-0.15}^{+0.24}, \Omega_{\Lambda}=0.74_{-0.24}^{+0.15}$ and $\Omega_{M}=0.40_{-0.26}^{+0.51}, \Omega_{\Lambda}=0.60_{-0.51}^{+0.26}$ from the $100 \mathrm{kpc}$ cut $\mathrm{X}$-ray and SZE samples, respectively, and $\Omega_{M}=0.40_{-0.20}^{+0.28}$, $\Omega_{\Lambda}=0.60_{-0.28}^{+0.20}$ and $\Omega_{M}=0.18_{-0.17}^{+0.27}, \Omega_{\Lambda}=0.82_{-0.27}^{+0.17}$ from the nonisothermal X-ray and SZE samples, respectively. Our results are consistent with standard $\Lambda$ CDM cosmology and inconsistent with $\Omega_{M}=1$ and $\Omega_{\Lambda}=0$. Interestingly, the systematic uncertainty for this method is negligible compared to the statistical uncertainty, as most sources of systematic uncertainty are not expected to introduce trends with redshift. Cosmological constraints using this method are therefore expected to improve rapidly as the cluster sample size increases with upcoming X-ray and SZE cluster surveys.

We are grateful to the late Leon van Speybroeck for compiling Chandra data for a large ensemble of clusters, and to his colleagues on the Chandra project as well, especially C. Jones and H. Tananbaum, who generously shared proprietary data with us. The support of the BIMA and OVRO staff over many years is also gratefully acknowledged, including J. R. Forster, C. Giovanine, R. Lawrence, S. Padin, R. Plambeck, S. Scott, and D. Woody. We thank C. Alexander, K. Coble, A. Cooray, L. Grego, G. Holder, J. Hughes, W. Holzapfel, A. Miller, J. Mohr, S. Patel, and P. Whitehouse for their contributions to the SZE instrumentation, observations, and analysis. We thank our referee K. Romer for her helpful comments in improving the manuscript. Finally, we thank A. Kravtsov for many useful discussions.

This work was supported by NASA LTSA grant NAG5-7985 and also in part by NSF grants PHY 01-14422 and AST 00-96913, the David and Lucile Packard Foundation, the McDonnell Foundation, and a MSFC director's discretionary award. Research at the Owens Valley Radio Observatory and the Berkeley-IllinoisMaryland Association array was supported by NSF grants AST 99-81546 and 02-28963. S. L. and D. N. acknowledge support from the NASA Graduate Student Researchers Program. D. N. is also supported by the Sherman Fairchild Postdoctoral Fellowship at Caltech.

\section{APPENDIX}

\section{SURFACE BRIGHTNESS PROFILES}

Figure 5 shows the X-ray surface brightness profiles and best-fit $100 \mathrm{kpc}$ cut models for the 38 clusters in our sample. 

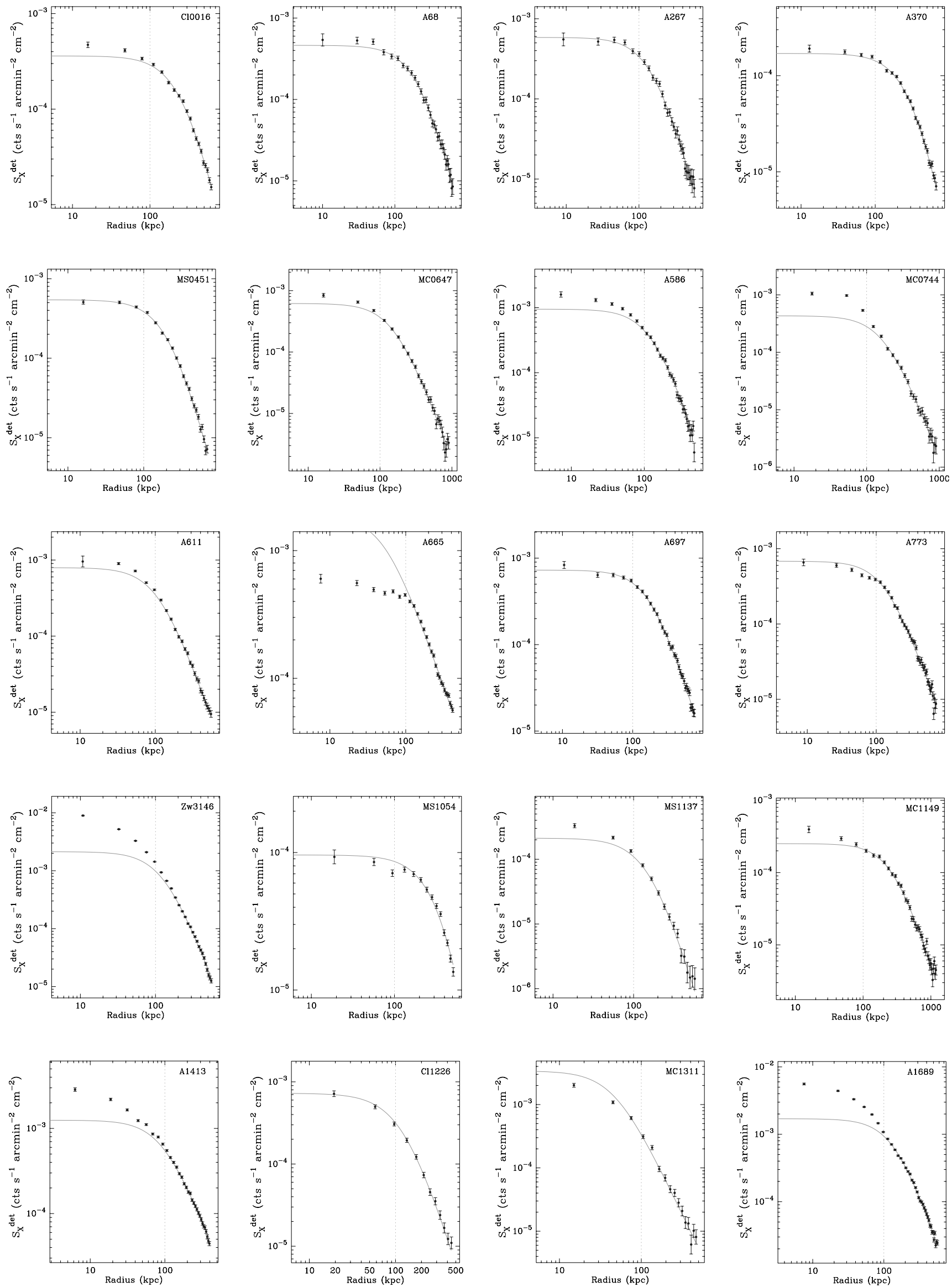

FIG. 5.-X-ray surface brightness radial profiles ( points) with best-fit $100 \mathrm{kpc}$ cut models (solid lines). Radii are calculated using a reasonable input cosmology of $\Omega_{M}=0.3, \Omega_{\Lambda}=0.7$, and $h=0.7$. The vertical dotted line is drawn at $100 \mathrm{kpc}$; data within this radius are not used in the fit. [See the electronic edition of the Journal for a color version of this figure.] 

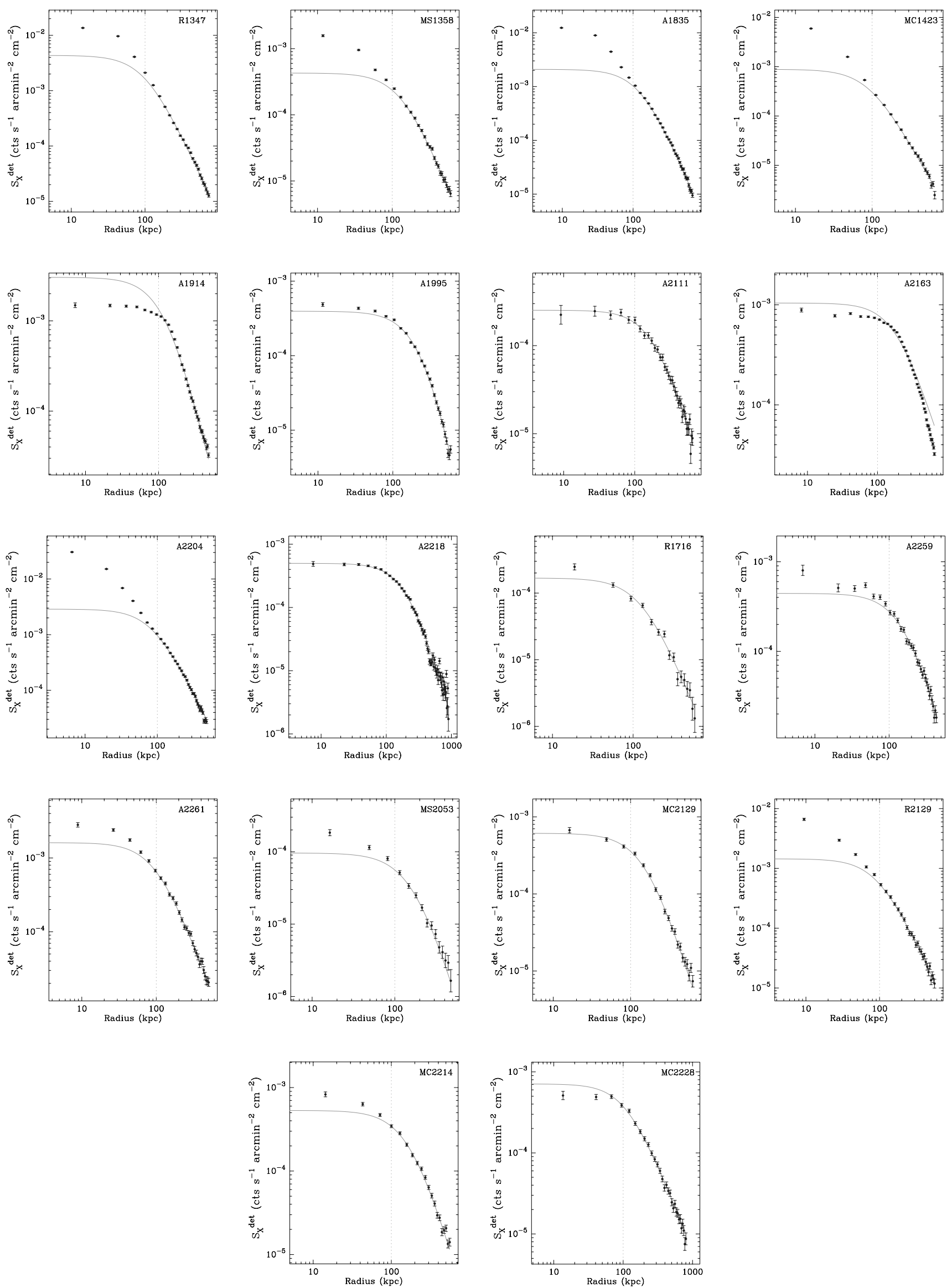

Fig. 5-Continued 
Afshordi, N., Lin, Y.-T., \& Sanderson, A. J. R. 2005, ApJ, 629, 1

Allen, S. W., Schmidt, R. W., Ebeling, H., Fabian, A. C., \& van Speybroeck, L. 2004, MNRAS, 353, 457

Allen, S. W., Schmidt, R. W., \& Fabian, A. C. 2002, MNRAS, 334, L11

Allen, S. W., et al. 1992, MNRAS, 259, 67

Arnaud, K. A. 1996, in ASP Conf. Ser. 101, Astronomical Data Analysis Software and Systems V, ed. G. H. Jacoby \& J. Barnes (San Francisco: ASP), 17

Bahcall, N. A., \& Fan, X. 1998, ApJ, 504, 1

Birkinshaw, M., Hughes, J. P., \& Arnaud, K. A. 1991, ApJ, 379, 466

Böhringer, H., et al. 2000, ApJS, 129, 435

Bonamente, M., Joy, M. K., Carlstrom, J. E., Reese, E. D., \& LaRoque, S. J. 2004, ApJ, 614, 56

Bonamente, M., Joy, M. K., LaRoque, S. J., Carlstrom, J. E., Reese, E. D., \& Dawson, K. S. 2006, ApJ, 647, 25

Borgani, S., et al. 2004, MNRAS, 348, 1078

Carlstrom, J. E., Holder, G. P., \& Reese, E. D. 2002, ARA\&A, 40, 643

Carlstrom, J. E., Joy, M., \& Grego, L. 1996, ApJ, 456, L75

Cash, W. 1979, ApJ, 228, 939

Cavaliere, A., \& Fusco-Femiano, R. 1976, A\&A, 49, 137 1978, A\&A, 70, 677

Colberg, J. M., White, S. D. M., MacFarland, T. J., Jenkins, A., Pearce, F. R., Frenk, C. S., Thomas, P. A., \& Couchman, H. M. P. 2000, MNRAS, 313, 229

Condon, J. J., Cotton, W. D., Greisen, E. W., Yin, Q. F., Perley, R. A., Taylor,

G. B., \& Broderick, J. J. 1998, AJ, 115, 1693

Cooray, A. R. 1998, A\&A, 339, 623

Cooray, A. R., Grego, L., Holzapfel, W. L., Joy, M., \& Carlstrom, J. E. 1998, AJ, 115, 1388

David, L. P., Jones, C., \& Forman, W. 1995, ApJ, 445, 578

Dickey, J. M., \& Lockman, F. J. 1990, ARA\&A, 28, 215

Donahue, M., Voit, G. M., Gioia, I., Lupino, G., Hughes, J. P., \& Stocke, J. T. 1998, ApJ, 502, 550

Donahue, M., Voit, G. M., Scharf, C. A., Gioia, I. M., Mullis, C. R., Hughes, J. P., \& Stocke, J. T. 1999, ApJ, 527, 525

Ebeling, H., Edge, A. C., Böhringer, H., Allen, S. W., Crawford, C. S., Fabian, A. C., Voges, W., \& Huchra, J. P. 1998, MNRAS, 301, 881

Ebeling, H., Jones, L. R., Fairley, B. W., Perlman, E., Scharf, C., \& Horner, D. 2001, ApJ, 548, L23

Elbaz, D., Arnaud, M., \& Böhringer, H. 1995, A\&A, 293, 337

Ettori, S., Dolag, K., Borgani, S., \& Murante, G. 2006, MNRAS, 365, 1021

Ettori, S., \& Fabian, A. C. 1999, MNRAS, 305, 834

Ettori, S., Tozzi, P., Borgani, S., \& Rosati, P. 2004, A\&A, 417, 13

Ettori, S., Tozzi, P., \& Rosati, P. 2003, A\&A, 398, 879

Evrard, A. E. 1997, MNRAS, 292, 289

Evrard, A. E., Metzler, C. A., \& Navarro, J. F. 1996, ApJ, 469, 494

Faltenbacher, A., Kravtsov, A. V., Nagai, D., \& Gottlöber, S. 2005, MNRAS, 358,139

Feldman, U. 1992, Phys. Scr. T, 46, 202

Freedman, W. L., et al. 2001, ApJ, 553, 47

Gioia, I. M., \& Luppino, G. A. 1994, ApJS, 94, 583

Gould, R. J. 1980, ApJ, 238, 1026

Grego, L., Carlstrom, J. E., Joy, M. K., Reese, E. D., Holder, G. P., Patel, S., Cooray, A. R., \& Holzapfel, W. L. 2000, ApJ, 539, 39

Grego, L., Carlstrom, J. E., Reese, E. D., Holder, G. P., Holzapfel, W. L., Joy, M. K., Mohr, J. J., \& Patel, S. 2001, ApJ, 552, 2

Henry, J. P., et al. 1997, AJ, 114, 1293

Inogamov, N. A., \& Sunyaev, R. A. 2003, Astron. Lett., 29, 791

Itoh, N., Kohyama, Y., \& Nozawa, S. 1998, ApJ, 502, 7

Jones, C., \& Forman, W. 1984, ApJ, 276, 38 1999, ApJ, 511, 65

Jonker, P. G., Campana, S., Steeghs, D., Torres, M. A. P., Galloway, D. K., Markwardt, C. B., Chakrabarty, D., \& Swank, J. 2005, MNRAS, 361, 511

Kneissl, R., Jones, M. E., Saunders, R., Eke, V. R., Lasenby, A. N., Grainge, K., \& Cotter, G. 2001, MNRAS, 328, 783

Kosowsky, A. 2003, NewA Rev., 47, 939

Kravtsov, A. V. 1999, Ph.D. thesis, New Mexico State Univ.

\section{EFERENCES}

Kravtsov, A. V., Klypin, A., \& Hoffman, Y. 2002, ApJ, 571, 563

Kravtsov, A. V., Nagai, D., \& Vikhlinin, A. A. 2005, ApJ, 625, 588

LaRoque, S. J. 2005, Ph.D. thesis, Univ. Chicago

LaRoque, S. J., Reese, E. D., Carlstrom, J. E., Holder, G., Holzapfel, W. L., Joy, M., \& Grego, L. 2002, preprint (astro-ph/0204134)

LaRoque, S. J., et al. 2003, ApJ, 583, 559

Luppino, G. A., \& Gioia, I. M. 1995, ApJ, 445, L77

Mathiesen, B. F., \& Evrard, A. E. 2001, ApJ, 546, 100

Mazzotta, P., Rasia, E., Moscardini, L., \& Tormen, G. 2004, MNRAS, 354, 10

Mewe, R., Gronenschild, E. H. B. M., \& van den Oord, G. H. J. 1985, A\&AS, 62, 197

Mohr, J. J., Evrard, A. E., Fabricant, D. G., \& Geller, M. J. 1995, ApJ, 447, 8

Mohr, J. J., Mathiesen, B., \& Evrard, A. E. 1999, ApJ, 517, 627

Myers, S. T., Baker, J. E., Readhead, A. C. S., Leitch, E. M., \& Herbig, T. 1997, ApJ, 485, 1

Nagai, D. 2006, ApJ, 650, 538

Nagai, D., Sulkanen, M. E., \& Evrard, A. E. 2000, MNRAS, 316, 120

Nagai, D., Vikhlinin, A., \& Kravtsov, A. V. 2006, ApJ, in press (astro-ph/ 0609247)

Navarro, J. F., Frenk, C. S., \& White, S. D. M. 1996, ApJ, 462, 563 1997, ApJ, 490, 493

Patel, S. K., et al. 2000, ApJ, 541, 37

Pearson, T. J., Shepherd, M. C., Taylor, G. B., \& Myers, S. T. 1994, BAAS, 26, 1318

Pen, U. 1997, NewA, 2, 309

Perlmutter, S., et al. 1999, ApJ, 517, 565

Piffaretti, R., Jetzer, P., \& Schindler, S. 2003, A\&A, 398, 41

Rasia, E., et al. 2006, MNRAS, 369, 2013

Raymond, J. C., \& Smith, B. W. 1977, ApJS, 35, 419

Reese, E. D., Carlstrom, J. E., Joy, M., Mohr, J. J., Grego, L., \& Holzapfel, W. L. 2002, ApJ, 581, 53

Reese, E. D., et al. 2000, ApJ, 533, 38

Riess, A. G., et al. 1998, AJ, 116, 1009

Romer, A. K., Viana, P. T. P., Liddle, A. R., \& Mann, R. G. 2001, ApJ, 547, 594

Rudy, D. J. 1987, Ph.D. thesis, California Inst. Technol.

Ruhl, J. E., et al. 2004, Proc. SPIE, 5498, 11

Sanderson, A. J. R., \& Ponman, T. J. 2003, MNRAS, 345, 1241

Sanderson, A. J. R., Ponman, T. J., Finoguenov, A., Lloyd-Davies, E. J., \& Markevitch, M. 2003, MNRAS, 340, 989

Sarazin, C. L. 1988, X-Ray Emission from Clusters of Galaxies (Cambridge: Cambridge Univ. Press)

Sasaki, S. 1996, PASJ, 48, L119

Sault, R. J., Teuben, P. J., \& Wright, M. C. H. 1995, in ASP Conf. Ser. 77, Astronomical Data Analysis Software and Systems IV, ed. R. A. Shaw, H. E. Payne, \& J. J. E. Hayes (San Francisco: ASP), 433

Schindler, S., et al. 1995, A\&A, 299, L9

Schuecker, P., Finoguenov, A., Miniati, F., Böhringer, H., \& Briel, U. G. 2004, A\&A, 426, 387

Scoville, N. Z., Carlstrom, J. E., Chandler, C. J., Phillips, J. A., Scott, S. L., Tilanus, R. P. J., \& Wang, Z. 1993, PASP, 105, 1482

Spergel, D. N., et al. 2006, ApJ, submitted (astro-ph/0603449)

Stocke, J. T., Morris, S. L., Gioia, I. M., Maccacaro, T., Schild, R., Wolter, A., Fleming, T. A., \& Henry, J. P. 1991, ApJS, 76, 813

Struble, M. F., \& Rood, H. J. 1999, ApJS, 125, 35

Sunyaev, R. A., \& Zel'dovich, Ya. B. 1970, Comments Astrophys. Space Phys., 2, 66

. 1972, Comments Astrophys. Space Phys., 4, 173

Vikhlinin, A. 2006, ApJ, 640, 710

Vikhlinin, A., Kravtsov, A., Forman, W., Jones, C., Markevitch, M., Murray, S. S., \& Van Speybroeck, L. 2006, ApJ, 640, 691

Watkins, R. 1997, MNRAS, 292, L59

White, S. D. M., Navarro, J. F., Evrard, A. E., \& Frenk, C. S. 1993, Nature, 366,429

Winkler, C., et al. 1995, A\&A, 302, 765 